Article

\title{
Evaluating the Effects of Steel Fibers on Mechanical Properties of Ultra-High Performance Concrete Using Artificial Neural Networks
}

\author{
Dechao Qu ${ }^{1}$, Xiaoping Cai ${ }^{1}$ and Wei Chang ${ }^{2, *}$ \\ 1 School of Transportation Science and Engineering, Harbin Institute of Technology, Harbin 150090, China; \\ qdc-1989@163.com (D.Q.); chaicp@Sina.com (X.C.) \\ 2 School of Civil Engineering, Harbin Institute of Technology, Harbin 150090, China \\ * Correspondence: changweihit@hit.edu.cn
}

Received: 9 June 2018; Accepted: 9 July 2018; Published: 11 July 2018

check for updates

Featured Application: This work can be utilized to predict the flexural strength and the compressive strength of ultra-high performance concrete (UHPC), determine the volume fraction of steel fibers in ultra-high performance steel fiber reinforced concrete (UHPFRC), and optimize the UHPFRC mixtures.

\begin{abstract}
Steel fibers enhance the flexural strength, the compressive strength and the ductility of untra-high performance concrete, predicting the flexural strength and the compressive strength of ultra-high performance steel fiber reinforced concrete (UHPFRC) accurately has significant influence on controlling steel fiber volume fraction and optimizing UHPFRC mix proportion. In this study, to evaluate the effects of steel fibers on the mechanical properties of UHPFRC, two artificial neural networks were developed in order to predict the flexural strength and the compressive strength of UHPFRC, respectively. 102 test data sets and 162 test data sets from literature were trained and tested to establish the flexural strength model and the compressive strength model, respectively. In these two models, the influential parameters, including the water to binder ratio, the diameter, the length, the aspect ratio, and the volume fraction of steel fibers, as well as the compressive strength and the flexural strength of concrete without fibers were investigated as the inputs, while the compressive strength and the flexural strength of UHPFRC were the outputs. The results show that the artificial neural network models predicted the compressive strength and flexural strength of UHPFRC accurately. Then, by comparing with existing analytical models, it was determined that the proposed models had high applicability and reliability with respect to predicting the compressive strength and the flexural strength of UHPFRC.
\end{abstract}

Keywords: artificial neural model; compressive strength; flexural strength; ultra-high performance concrete; steel fiber

\section{Introduction}

In ultra-high performance concrete (UHPC) mixture design, the compressive strength, and the flexural strength are two key mechanic parameters to evaluate the strength and the ductility of materials. Because UHPC is brittle, the steel fibers with high tensile strength and high ultimate elongation, are always uniformly dispersed in UHPC to increase the ductility and the strength of concrete. The UHPC with steel fibers is also called ultra-high performance steel fiber reinforced concrete (UHPFRC). The steel fibers in UHPFRC improve the cohesive forces between fibers and matrix, change the granular skeleton, and increase the anchorage length between fibers and the surrounding matrix [1,2]. Besides, steel fibers bridge cracks and retard the propagation to increase 
the strength and the ductility of UHPFRC [3]. Unfortunately, too many steel fibers lead to fibers inter-wrap and interlock with each other, affecting the workability of UHPFRC, to reduce the strength of UHPFRC [4]. Furthermore, steel fibers are expensive and numerous steel fibers added to the UHPFRC cost too much. Thus, predicting the compressive strength and the flexural strength of UHPFRC accurately can optimize mix proportion, control the volume fraction of steel fibers, and decrease the costs of UHPFRC. However, evaluating the flexural strength and the compressive strength of the UHPFRC is a huge challenge due to the complex composite behavior caused by the properties steel fibers (diameter $(D)$, length $(L)$, aspect ratio $(A R)$, and volume fraction $(V F)$ ) and concrete matrix (water to binder ratio $(W / B)$ and concrete strength without fibers).

Nowadays, the contribution of cement-based materials to sustainability is a topic of study [5-8] and the performance of several additions in cement-based materials, such as silica fume, fly ash, the water to cement ratio, and so on, has been analyzed, which could be also suitable for being used in high performance concrete. This fact also makes it necessary to consider their influence on the mechanical properties of these new concretes. In the past decades, different experimental and theoretical investigations [9-30] have been carried out on the compressive strength and the flexural strength of UHPFRC. Several analytical models have been constructed to predict the compressive strength and the flexural strength of UHPFRC by proposing a series of assumptions about the mechanism and developing the equilibrium equations. Furthermore, several empirical models [31-36], including those used in the design codes, have been established based on the experimental studies to predict the compressive strength and the flexural strength of UHPFRC. Additionally, the Chinese standard JGJ/T 221 [31] only proposes the empirical model to predict the flexural strength of steel fiber reinforced concrete. In general, these models mainly depend on the diameter, the length, and the volume fraction of steel fibers and the compressive/flexural strength of UHPFRC without fibers. However, the experimental data used to establish the empirical formula is limited, and most of them mainly focus on the volume fraction of steel fibers and does not consider other parameters. Thus, when new test data is available, the predictive accuracy and reliability of those empirical models have to be re-evaluated.

In recent years, the artificial intelligence techniques of artificial neural networks (ANNs) have been widely adopted to solve many engineering complex problems due to its high accuracy and adaptability. The ANN model has been successfully used to evaluate the early-age autogenous shrinkage of concrete [37], estimate the compressive strength of FRP-confined (FRP denotes fiber reinforced polymer) concrete circular columns [38], optimize the mix compositions of steel fiber-reinforced concrete [39], and so on. However, very few studies have been conducted on the application of ANNs to predict the compressive strength and the flexural strength of UHPFRC. A. Jayaranjini presented an ANN model to predict the compressive strength of concrete containing industrial byproducts at the age of $28,56,90$, and 120 days [40]. F. Altun et al. estimated the compressive strength of steel fiber reinforced lightweight concrete by ANN [41]. Moreover, D. Zealakshmi et al. developed an ANN model to predict the flexural performance of confined hybrid fiber reinforced high strength concrete beam with varying the volume fraction of steel and polypropylene fibers [42]. As for predicting the effects of steel fibers on the compressive strength and the flexural strength of UHPFRC, because of the high adaptability and high accuracy, the ANN models are suitable for evaluating the mechanical behavior of UHPFRC.

The objective of this study is to develop two ANN models to predict the compressive strength and the flexural strength of UHPFRC. To achieve this purpose, 162 compressive strength data sets and 102 flexural strength data sets were collected from published literature to develop models and to evaluate the effects of steel fibers on the compressive strength and the flexural strength of UHPFRC, respectively. Finally, the proposed models were compared with several analytical models to evaluate their reliability and predictability. Furthermore, the ANN models can be utilized to predict the flexural strength and the compressive strength of UHPC, determine the volume fraction of steel fibers in UHPFRC, and optimize the UHPFRC mixtures. 


\section{Artificial Neural Network Approach}

Artificial neural network (ANN) is a machine learning algorithm that attempted to simulate the nervous system of human to process experimental data by classifying, clustering, regressing, and predicting [43]. Multi-layer feed-forward perception network is one of a typical ANN, the topological structure of this network has an input layer, one or more hidden layers, and an output layer, in which the numerous neurons are distributed into layers (in Figure 1). In the network, all of the neurons in each layer are connected to the next layer, and there is no connection among neurons in the same layer. The conversion between the input and the output layer is accomplished by adjusting the weights and thresholds among layers. If the problem can be learned, a set of weights will be stable and a good result will be obtained.

For multi-layer feed forward networks, the back propagation neural network is one of the most rudimentary and most important neural networks using nonlinear training methods. The training method of the back propagation network is based on supervised learning, which minimizes the errors between the calculated and experimental values by adjusting the weights in a small amounts each time $[40,41]$.

The training processes of a back propagation network have two stages: the forward stage and the backward stage. The forward stage calculates the network outputs through the given initial connection weights and the input data. In the process, the input data is passed from the input layer to the hidden layer. Then, the neurons in the hidden layer calculate a weighted sum of the input data, processes the sum by using an activation function, and finally pass the activation results to the output layer. The weighted sum of the input data can be calculated by Equation (1) [43-46].

$$
n e t_{j}=\sum \omega_{i j} x_{i}+b_{j}
$$

where $n e t_{j}$ is the weighted sum of the $j_{t h}$ neuron received from the lower layer with $n$ neurons, $\omega_{i j}$ is the weight between the $i_{t h}$ neuron in the lower layer and the $j_{t h}$ neuron in the upper layer, $x_{i}$ is the output of the $i_{t h}$ neuron in the lower layer, and $b_{j}$ is the bias of the $j_{t h}$ layer in the upper layer. The activation function in back propagation network generally is Sigmoid function or Linear function. The Sigmoid function can be expressed in Equation (2).

$$
\begin{aligned}
\text { net }_{j} & =\sum \omega_{i j} x_{i}+b_{j} \\
o_{j} & =\frac{1}{1+e^{-n e t_{j}}}
\end{aligned}
$$

where $o_{j}$ is the output of the $j_{t h}$ neuron in the upper layer.

In the backward stage, the errors between the calculated outputs and the experimental results are calculated, and the errors are passed back to the network to modify the connection weights and bias. This process is repeated until the errors reach an acceptable level. The adjusted value of the weights can be summarized in Equation (4):

$$
\Delta \omega_{\mathrm{ij}}(n)=\eta \times \delta_{J}^{j} \times o_{I}^{i}
$$

where $\Delta \omega_{i j}$ is the $n_{t h}$ value of weights adjustment between the $i_{t h}$ neuron in the lower layer and the $j_{t h}$ neuron in the upper layer, $\eta$ is the training rate, $\delta_{J}^{j}$ is the local gradient of the $j_{t h}$ layer, and $o_{I}^{i}$ is the output of the input neurons.

In this study, the training method of the ANN models uses the LM (Levenberg-Marquardt) algorithm, because the LM algorithm combines the advantages of the Quasi-Newton algorithm and the Steepest Descent Back-propagation, which is suitable for non-linear least square problems and curve fitting [45]. 


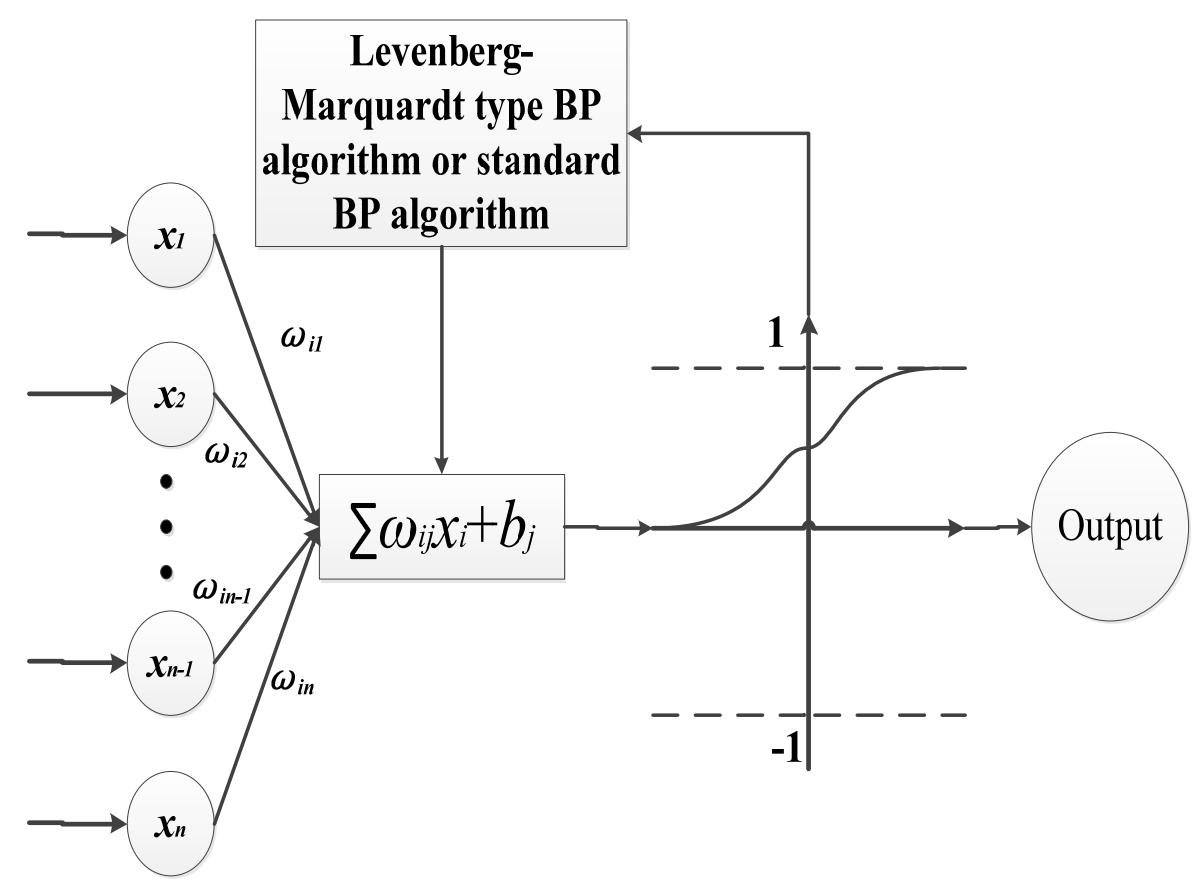

Figure 1. System of typical artificial neural networks (ANN) model. A typical ANN model has input, sum function, log-sigmoid activation function, and output.

\section{Database and Models}

\subsection{Data Collection}

To explore the effects of steel fibers on the compressive strength and the flexural strength of UHPFRC, a reliable database was established. 162-group compressive strength experimental data and 102-group flexural strength experimental data were gathered from the literature [9-30] (Tables 1 and 2).

The specimens for compressive strength testing varies in shapes and sizes, due to lack of proper standards. According to GB/T 31387 [47], cube specimen of $100 \times 100 \times 100 \mathrm{~mm}$ is considered as standard for homogeneity of data, The test specimens were converted to standard cube compressive strength by the correlation techniques suggested by researchers [48-51] and the equations are as follows.

$$
\begin{gathered}
f_{c u, 100}=0.959 f_{c u, 70.7} \\
f_{c}^{\prime}=0.845 f_{c u, 70.7} \\
f_{c u, 150}=0.91 f_{c u, 100}+3.62 \\
f_{c u, 150}=f_{c y l i n, 100 \times 200}+6.41 \\
f_{c y l i n, 50 \times 100}=1.07 f_{c y l i n, 100 \times 200}
\end{gathered}
$$

where, $f_{c u, 70.7} f_{c u, 100}$, and $f_{c u, 150}$ are the compressive strength of $70.7 \mathrm{~mm}$ cube, $100 \mathrm{~mm}$ cube, and $150 \mathrm{~mm}$ cube, respectively; $f_{c y l i n, 50 \times 100}$ and $f_{c y l i n, 100 \times 200}$ are the compressive strength of $50 \times 100 \mathrm{~mm}$ cylinder and $100 \times 200 \mathrm{~mm}$ cylinder, respectively; $f_{c}^{\prime}$ is the axis compressive strength of UHPFRC, the size effects are not obvious in axis compressive strength. 
Table 1. Experimental Data used for establishing Compressive Strength Artificial neyral network (ANN) model.

\begin{tabular}{|c|c|c|c|c|c|c|c|c|c|}
\hline \multirow{2}{*}{ No. } & \multirow{2}{*}{$\begin{array}{l}\text { Specimen } \\
\text { Dimension }\end{array}$} & \multirow{2}{*}{ *W/B } & \multicolumn{4}{|c|}{ Steel Fiber } & \multirow{2}{*}{ * PCS } & \multirow{2}{*}{$* * C S$} & \multirow{2}{*}{ Reference } \\
\hline & & & ${ }^{*} D / \mathrm{mm}$ & $* L / \mathrm{mm}$ & $* A R$ & $* V F / \%$ & & & \\
\hline 1 & \multirow{16}{*}{$\begin{aligned} & \text { Cylinder } \\
& \mathrm{D} \times \mathrm{H} 100 \mathrm{~mm} \times \\
& 200 \mathrm{~mm}\end{aligned}$} & 0.30 & 0.20 & 13.00 & 65.00 & 0.00 & 94.17 & 94.17 & [9] \\
\hline 2 & & 0.30 & 0.20 & 13.00 & 65.00 & 0.50 & 94.17 & 96.13 & [9] \\
\hline 3 & & 0.30 & 0.20 & 13.00 & 65.00 & 1.00 & 94.17 & 98.90 & [9] \\
\hline 4 & & 0.30 & 0.20 & 13.00 & 65.00 & 2.00 & 94.17 & 103.05 & [9] \\
\hline 5 & & 0.25 & 0.20 & 13.00 & 65.00 & 0.00 & 103.05 & 120.17 & [9] \\
\hline 6 & & 0.25 & 0.20 & 13.00 & 65.00 & 0.50 & 103.05 & 122.90 & [9] \\
\hline 7 & & 0.25 & 0.20 & 13.00 & 65.00 & 1.00 & 103.05 & 127.80 & [9] \\
\hline 8 & & 0.25 & 0.20 & 13.00 & 65.00 & 2.00 & 103.05 & 133.19 & [9] \\
\hline 9 & & 0.20 & 0.20 & 13.00 & 65.00 & 0.00 & 168.27 & 168.27 & [9] \\
\hline 10 & & 0.20 & 0.20 & 13.00 & 65.00 & 0.50 & 168.27 & 174.27 & [9] \\
\hline 11 & & 0.20 & 0.20 & 13.00 & 65.00 & 1.00 & 168.27 & 179.28 & [9] \\
\hline 12 & & 0.20 & 0.20 & 13.00 & 65.00 & 2.00 & 168.27 & 182.31 & [9] \\
\hline 13 & & 0.17 & 0.20 & 13.00 & 65.00 & 0.00 & 220.98 & 220.98 & [9] \\
\hline 14 & & 0.17 & 0.20 & 13.00 & 65.00 & 0.50 & 220.98 & 225.83 & [9] \\
\hline 15 & & 0.17 & 0.20 & 13.00 & 65.00 & 1.00 & 220.98 & 234.15 & [9] \\
\hline 16 & & 0.17 & 0.20 & 13.00 & 65.00 & 2.00 & 220.98 & 240.75 & [9] \\
\hline 17 & \multirow{6}{*}{$\begin{array}{c}\text { Cube } \\
70.7 \mathrm{~mm} \times \\
70.7 \mathrm{~mm} \times \\
70.7 \mathrm{~mm}\end{array}$} & 0.16 & 0.16 & 13.00 & 81.25 & 2.50 & 126.59 & 172.59 & [10] \\
\hline 18 & & 0.16 & 0.16 & 13.00 & 81.25 & 2.00 & 126.59 & 163.31 & [10] \\
\hline 19 & & 0.16 & 0.16 & 13.00 & 81.25 & 0.00 & 126.59 & 126.59 & [10] \\
\hline 20 & & 0.16 & 0.16 & 6.00 & 37.50 & 2.50 & 126.59 & 171.37 & [10] \\
\hline 21 & & 0.16 & 0.16 & 6.00 & 37.50 & 2.00 & 126.59 & 157.28 & [10] \\
\hline 22 & & 0.16 & 0.16 & 6.00 & 37.50 & 0.00 & 126.59 & 126.59 & [10] \\
\hline 23 & \multirow{3}{*}{$\begin{array}{c}\text { Cube } \\
100 \mathrm{~mm} \times 100 \mathrm{~mm} \\
\times 100 \mathrm{~mm}\end{array}$} & 0.24 & 0.20 & 20.00 & 100.00 & 0.00 & 111.00 & 111.00 & [11] \\
\hline 24 & & 0.24 & 0.20 & 20.00 & 100.00 & 1.00 & 111.00 & 101.00 & [11] \\
\hline 25 & & 0.24 & 0.20 & 20.00 & 100.00 & 2.00 & 111.00 & 112.00 & [11] \\
\hline 26 & \multirow{4}{*}{$\begin{array}{c}\text { Cylinder } \\
\mathrm{D} \times \mathrm{H} 100 \mathrm{~mm} \times \\
200 \mathrm{~mm}\end{array}$} & 0.14 & 0.50 & 30.00 & 60.00 & 0.00 & 141.72 & 141.72 & [12] \\
\hline 27 & & 0.14 & 0.50 & 30.00 & 60.00 & 1.00 & 141.72 & 146.99 & [12] \\
\hline 28 & & 0.14 & 0.50 & 30.00 & 60.00 & 2.00 & 141.72 & 153.57 & [12] \\
\hline 29 & & 0.15 & 0.50 & 30.00 & 60.00 & 3.00 & 141.72 & 154.33 & [12] \\
\hline 30 & \multirow{12}{*}{$\begin{array}{c}\text { Cube } \\
100 \mathrm{~mm} \times 100 \mathrm{~mm} \\
\times 100 \mathrm{~mm}\end{array}$} & 0.18 & 0.24 & 13.5 & 56.25 & 0.00 & 129.92 & 129.92 & [13] \\
\hline 31 & & 0.18 & 0.24 & 13.5 & 56.25 & 1.00 & 129.92 & 135.89 & [13] \\
\hline 32 & & 0.18 & 0.24 & 13.5 & 56.25 & 2.00 & 129.92 & 142.46 & [13] \\
\hline 33 & & 0.18 & 0.24 & 13.5 & 56.25 & 3.00 & 129.92 & 155.09 & [13] \\
\hline 34 & & 0.18 & 0.24 & 13.5 & 56.25 & 0.00 & 133.88 & 133.88 & [13] \\
\hline 35 & & 0.18 & 0.24 & 13.5 & 56.25 & 1.00 & 133.88 & 141.06 & [13] \\
\hline 36 & & 0.18 & 0.24 & 13.5 & 56.25 & 2.00 & 133.88 & 150.62 & [13] \\
\hline 37 & & 0.18 & 0.24 & 13.5 & 56.25 & 3.00 & 133.88 & 160.43 & [13] \\
\hline 38 & & 0.18 & 0.24 & 13.5 & 56.25 & 0.00 & 142.38 & 142.38 & [13] \\
\hline 39 & & 0.18 & 0.24 & 13.5 & 56.25 & 1.00 & 142.38 & 153.25 & [13] \\
\hline 40 & & 0.18 & 0.24 & 13.5 & 56.25 & 2.00 & 142.38 & 169.01 & [13] \\
\hline 41 & & 0.18 & 0.24 & 13.5 & 56.25 & 3.00 & 142.38 & 172.52 & [13] \\
\hline 42 & \multirow{5}{*}{$\begin{array}{c}\text { Cube } \\
70.7 \mathrm{~mm} \times \\
70.7 \mathrm{~mm} \times \\
70.7 \mathrm{~mm}\end{array}$} & 0.20 & 0.20 & 13.00 & 65.00 & 0.00 & 88.49 & 88.49 & [14] \\
\hline 43 & & 0.20 & 0.20 & 13.00 & 65.00 & 0.50 & 88.49 & 105.37 & [14] \\
\hline 44 & & 0.20 & 0.20 & 13.00 & 65.00 & 1.50 & 88.49 & 112.29 & [14] \\
\hline 45 & & 0.20 & 0.20 & 13.00 & 65.00 & 2.50 & 88.49 & 128.91 & {$[14]$} \\
\hline 46 & & 0.20 & 0.20 & 13.00 & 65.00 & 3.50 & 88.49 & 132.32 & [14] \\
\hline 47 & \multirow{5}{*}{$\begin{array}{c}\text { Prism } \\
40 \mathrm{~mm} \times 40 \mathrm{~mm} \times \\
160 \mathrm{~mm}\end{array}$} & 0.24 & 0.25 & 13.00 & 52.00 & 0.00 & 115.86 & 115.86 & [15] \\
\hline 48 & & 0.24 & 0.25 & 13.00 & 52.00 & 1.00 & 115.86 & 125.15 & [15] \\
\hline 49 & & 0.24 & 0.25 & 13.00 & 52.00 & 1.50 & 115.86 & 126.40 & [15] \\
\hline 50 & & 0.24 & 0.25 & 13.00 & 52.00 & 2.00 & 115.86 & 132.69 & [15] \\
\hline 51 & & 0.24 & 0.25 & 13.00 & 52.00 & 2.50 & 115.86 & 137.32 & [15] \\
\hline 52 & \multirow{4}{*}{$\begin{array}{c}\text { Cube } \\
100 \mathrm{~mm} \times 100 \mathrm{~mm} \\
\times 100 \mathrm{~mm}\end{array}$} & 0.23 & 0.20 & 13.00 & 65.00 & 2.00 & 145.55 & 161.30 & [16] \\
\hline 53 & & 0.23 & 0.20 & 13.00 & 65.00 & 4.00 & 145.55 & 186.30 & [16] \\
\hline 54 & & 0.23 & 0.20 & 13.00 & 65.00 & 5.00 & 145.55 & 201.30 & [16] \\
\hline 55 & & 0.23 & 0.20 & 13.00 & 65.00 & 0.00 & 145.55 & 145.55 & [16] \\
\hline
\end{tabular}


Table 1. Cont.

\begin{tabular}{|c|c|c|c|c|c|c|c|c|c|}
\hline \multirow[b]{2}{*}{ No. } & \multirow{2}{*}{$\begin{array}{l}\text { Specimen } \\
\text { Dimension }\end{array}$} & \multirow{2}{*}{${ }^{*} \mathbf{W} / \mathbf{B}$} & \multicolumn{4}{|c|}{ Steel Fiber } & \multirow[b]{2}{*}{${ }^{*} P C S$} & \multirow[b]{2}{*}{$* * C S$} & \multirow{2}{*}{ Reference } \\
\hline & & & ${ }^{*} \mathrm{D} / \mathrm{mm}$ & ${ }^{*} \mathrm{~L} / \mathrm{mm}$ & ${ }^{*} A R$ & ${ }^{*} V F / \%$ & & & \\
\hline 56 & Cube & 0.20 & 0.22 & 13.00 & 59.09 & 0.00 & 72.15 & 72.15 & [17] \\
\hline 57 & $70.7 \mathrm{~mm} \times$ & 0.20 & 0.22 & 13.00 & 59.09 & 1.00 & 72.15 & 125.10 & [17] \\
\hline 58 & $70.7 \mathrm{~mm} \times$ & 0.20 & 0.22 & 13.00 & 59.09 & 2.00 & 72.15 & 147.86 & [17] \\
\hline 59 & $70.7 \mathrm{~mm}$ & 0.20 & 0.22 & 13.00 & 59.09 & 3.00 & 72.15 & 160.17 & [17] \\
\hline 60 & \multirow{5}{*}{$\begin{array}{c}\text { Prism } \\
40 \mathrm{~mm} \times 40 \mathrm{~mm} \times \\
160 \mathrm{~mm}\end{array}$} & 0.25 & 0.24 & 13.00 & 54.17 & 0.00 & 108.04 & 108.04 & [18] \\
\hline 61 & & 0.16 & 0.24 & 13.00 & 54.17 & 0.50 & 108.04 & 113.39 & [18] \\
\hline 62 & & 0.16 & 0.24 & 13.00 & 54.17 & 1.00 & 108.04 & 117.40 & [18] \\
\hline 63 & & 0.16 & 0.24 & 13.00 & 54.17 & 1.50 & 108.04 & 135.66 & [18] \\
\hline 64 & & 0.16 & 0.24 & 13.00 & 54.17 & 2.00 & 108.04 & 167.67 & [18] \\
\hline 65 & \multirow{4}{*}{$\begin{array}{c}\text { Prism } \\
40 \mathrm{~mm} \times 40 \mathrm{~mm} \times \\
160 \mathrm{~mm}\end{array}$} & 0.17 & 0.16 & 9.00 & 56.25 & 0.00 & 147.54 & 147.54 & [19] \\
\hline 66 & & 0.19 & 0.16 & 9.00 & 56.25 & 1.10 & 147.54 & 156.62 & [19] \\
\hline 67 & & 0.19 & 0.16 & 9.00 & 56.25 & 2.10 & 147.54 & 194.07 & [19] \\
\hline 68 & & 0.19 & 0.16 & 9.00 & 56.25 & 4.20 & 147.54 & 224.71 & [19] \\
\hline 69 & \multirow{4}{*}{$\begin{array}{c}\text { Cylinder } \\
\mathrm{D} \times \mathrm{H} 50 \mathrm{~mm} \times \\
100 \mathrm{~mm}\end{array}$} & 0.19 & 0.22 & 13.00 & 59.09 & 0.00 & 105.92 & 105.92 & [20] \\
\hline 70 & & 0.19 & 0.22 & 13.00 & 59.09 & 1.00 & 105.92 & 141.86 & [20] \\
\hline 71 & & 0.19 & 0.22 & 13.00 & 59.09 & 2.00 & 105.92 & 179.88 & [20] \\
\hline 72 & & 0.19 & 0.22 & 13.00 & 59.09 & 4.00 & 105.92 & 247.41 & [20] \\
\hline 73 & \multirow{13}{*}{$\begin{array}{c}\text { Prism } \\
40 \mathrm{~mm} \times 40 \mathrm{~mm} \times \\
160 \mathrm{~mm}\end{array}$} & 0.20 & 0.22 & 13.00 & 59.09 & 0.00 & 162.86 & 162.86 & [21] \\
\hline 74 & & 0.20 & 0.22 & 13.00 & 59.09 & 0.50 & 162.86 & 182.61 & [21] \\
\hline 75 & & 0.20 & 0.22 & 13.00 & 59.09 & 1.00 & 162.86 & 188.70 & [21] \\
\hline 76 & & 0.20 & 0.22 & 13.00 & 59.09 & 1.50 & 162.86 & 208.61 & [21] \\
\hline 77 & & 0.20 & 0.22 & 13.00 & 59.09 & 2.00 & 162.86 & 215.58 & [21] \\
\hline 78 & & 0.20 & 0.22 & 13.00 & 59.09 & 2.50 & 162.86 & 221.88 & [21] \\
\hline 79 & & 0.20 & 0.22 & 13.00 & 59.09 & 3.00 & 162.86 & 224.94 & [21] \\
\hline 80 & & 0.20 & 0.22 & 13.00 & 59.09 & 3.50 & 162.86 & 229.25 & [21] \\
\hline 81 & & 0.20 & 0.65 & 25.00 & 38.46 & 0.00 & 162.86 & 162.86 & [21] \\
\hline 82 & & 0.20 & 0.65 & 25.00 & 38.46 & 1.50 & 162.86 & 174.08 & [21] \\
\hline 83 & & 0.20 & 0.65 & 25.00 & 38.46 & 2.00 & 162.86 & 182.15 & [21] \\
\hline 84 & & 0.20 & 0.65 & 25.00 & 38.46 & 2.50 & 162.86 & 190.97 & [21] \\
\hline 85 & & 0.20 & 0.65 & 25.00 & 38.46 & 3.00 & 162.86 & 195.61 & [21] \\
\hline 86 & \multirow{5}{*}{$\begin{array}{c}\text { Cube } \\
100 \mathrm{~mm} \times 100 \mathrm{~mm} \\
\times 100 \mathrm{~mm}\end{array}$} & 0.17 & 0.22 & 14.00 & 63.64 & 0.00 & 88.68 & 88.68 & [22] \\
\hline 87 & & 0.17 & 0.22 & 14.00 & 63.64 & 0.50 & 88.68 & 102.52 & [22] \\
\hline 88 & & 0.17 & 0.22 & 14.00 & 63.64 & 1.00 & 88.68 & 127.00 & [22] \\
\hline 89 & & 0.17 & 0.22 & 14.00 & 63.64 & 1.50 & 88.68 & 130.02 & [22] \\
\hline 90 & & 0.17 & 0.22 & 14.00 & 63.64 & 2.00 & 88.68 & 142.87 & [22] \\
\hline 91 & \multirow{6}{*}{$\begin{array}{c}\text { Cube } \\
100 \mathrm{~mm} \times 100 \mathrm{~mm} \\
\times 100 \mathrm{~mm}\end{array}$} & 0.17 & 0.15 & 13.00 & 86.67 & 0.00 & 107.60 & 107.60 & [23] \\
\hline 92 & & 0.17 & 0.15 & 13.00 & 86.67 & 1.00 & 107.60 & 125.20 & [23] \\
\hline 93 & & 0.17 & 0.15 & 13.00 & 86.67 & 2.00 & 107.60 & 140.90 & [23] \\
\hline 94 & & 0.17 & 0.15 & 13.00 & 86.67 & 0.00 & 107.60 & 131.80 & [23] \\
\hline 95 & & 0.17 & 0.15 & 13.00 & 86.67 & 1.00 & 107.60 & 179.70 & [23] \\
\hline 96 & & 0.17 & 0.15 & 13.00 & 86.67 & 2.00 & 107.60 & 211.80 & [23] \\
\hline 97 & \multirow{14}{*}{$\begin{array}{c}\text { Prism } \\
100 \mathrm{~mm} \times 100 \mathrm{~mm} \\
\times 400 \mathrm{~mm}\end{array}$} & 0.22 & 0.23 & 13.00 & 57.78 & 0.00 & 107.23 & 107.23 & [24] \\
\hline 98 & & 0.22 & 0.23 & 13.00 & 57.78 & 0.50 & 107.23 & 119.58 & [24] \\
\hline 99 & & 0.22 & 0.23 & 13.00 & 57.78 & 1.00 & 107.23 & 104.78 & [24] \\
\hline 100 & & 0.22 & 0.23 & 13.00 & 57.78 & 2.00 & 107.23 & 105.74 & [24] \\
\hline 101 & & 0.22 & 0.23 & 13.00 & 57.78 & 3.00 & 107.23 & 104.68 & [24] \\
\hline 102 & & 0.22 & 0.23 & 13.00 & 57.78 & 0.00 & 147.10 & 147.10 & [24] \\
\hline 103 & & 0.22 & 0.23 & 13.00 & 57.78 & 0.50 & 147.10 & 161.57 & [24] \\
\hline 104 & & 0.22 & 0.23 & 13.00 & 57.78 & 1.00 & 147.10 & 163.59 & [24] \\
\hline 105 & & 0.22 & 0.23 & 13.00 & 57.78 & 2.00 & 147.10 & 159.21 & [24] \\
\hline 106 & & 0.22 & 0.23 & 13.00 & 57.78 & 3.00 & 147.10 & 151.94 & [24] \\
\hline 107 & & 0.22 & 0.23 & 13.00 & 57.78 & 0.00 & 160.52 & 160.52 & [24] \\
\hline 108 & & 0.22 & 0.23 & 13.00 & 57.78 & 1.00 & 160.52 & 165.13 & [24] \\
\hline 109 & & 0.22 & 0.23 & 13.00 & 57.78 & 2.00 & 160.52 & 166.03 & [24] \\
\hline 110 & & 0.22 & 0.23 & 13.00 & 57.78 & 3.00 & 160.52 & 167.99 & [24] \\
\hline
\end{tabular}


Table 1. Cont.

\begin{tabular}{|c|c|c|c|c|c|c|c|c|c|}
\hline No. & $\begin{array}{l}\text { Specimen } \\
\text { Dimension }\end{array}$ & * W/B & \multicolumn{4}{|c|}{ Steel Fiber } & $* P C S$ & $* * C S$ & Reference \\
\hline 111 & \multirow{8}{*}{$\begin{array}{c}\text { Prism } \\
100 \mathrm{~mm} \times 100 \mathrm{~mm} \\
\times 400 \mathrm{~mm}\end{array}$} & 0.16 & 0.22 & 13.50 & 61.36 & 0.00 & 125.80 & 125.80 & [25] \\
\hline 113 & & 0.16 & 0.22 & 13.50 & 61.36 & 3.00 & 125.80 & 150.30 & [25] \\
\hline 114 & & 0.16 & 0.22 & 13.50 & 61.36 & 4.00 & 125.80 & 152.20 & [25] \\
\hline 117 & & 0.16 & 0.22 & 13.50 & 61.36 & 3.00 & 128.60 & 154.70 & [25] \\
\hline 118 & & 0.16 & 0.22 & 13.50 & 61.36 & 4.00 & 128.60 & 156.80 & [25] \\
\hline 119 & & 0.16 & 0.22 & 13.50 & 61.36 & 0.00 & 134.90 & 134.90 & [25] \\
\hline 120 & & 0.16 & 0.22 & 13.50 & 61.36 & 2.00 & 134.90 & 156.90 & [25] \\
\hline 121 & & 0.16 & 0.22 & 13.50 & 61.36 & 3.00 & 134.90 & 162.30 & [25] \\
\hline 125 & \multirow{11}{*}{$\begin{array}{c}\text { Cube } \\
100 \mathrm{~mm} \times 100 \mathrm{~mm} \\
\times 100 \mathrm{~mm}\end{array}$} & 0.20 & 0.25 & 14.00 & 56.00 & 2.00 & 101.70 & 125.20 & [26] \\
\hline 126 & & 0.20 & 0.25 & 14.00 & 56.00 & 3.50 & 101.70 & 133.60 & [26] \\
\hline 127 & & 0.20 & 0.25 & 14.00 & 56.00 & 5.00 & 101.70 & 120.30 & [26] \\
\hline 128 & & 0.20 & 0.25 & 14.00 & 56.00 & 0.00 & 92.50 & 92.50 & [26] \\
\hline 129 & & 0.20 & 0.25 & 14.00 & 56.00 & 1.00 & 92.50 & 102.10 & [26] \\
\hline 130 & & 0.20 & 0.25 & 14.00 & 56.00 & 2.00 & 92.50 & 115.80 & [26] \\
\hline 131 & & 0.20 & 0.25 & 14.00 & 56.00 & 3.50 & 92.50 & 112.30 & [26] \\
\hline 132 & & 0.20 & 0.25 & 14.00 & 56.00 & 5.00 & 92.50 & 106.60 & [26] \\
\hline 133 & & 0.20 & 0.25 & 14.00 & 56.00 & 0.00 & 113.40 & 113.40 & [26] \\
\hline 134 & & 0.20 & 0.25 & 14.00 & 56.00 & 1.00 & 113.40 & 121.20 & [26] \\
\hline 135 & & 0.20 & 0.25 & 14.00 & 56.00 & 2.00 & 113.40 & 132.70 & [26] \\
\hline 143 & \multirow{5}{*}{$\begin{array}{c}\text { Cube } \\
100 \mathrm{~mm} \times 100 \mathrm{~mm} \\
\times 100 \mathrm{~mm}\end{array}$} & 0.14 & 0.22 & 13.00 & 59.09 & 0.00 & 108.72 & 108.72 & [27] \\
\hline 144 & & 0.14 & 0.22 & 13.00 & 59.09 & 0.50 & 108.72 & 121.36 & [27] \\
\hline 145 & & 0.14 & 0.22 & 13.00 & 59.09 & 1.00 & 108.72 & 136.97 & [27] \\
\hline 146 & & 0.14 & 0.22 & 13.00 & 59.09 & 1.50 & 108.72 & 152.19 & [27] \\
\hline 147 & & 0.14 & 0.22 & 13.00 & 59.09 & 2.00 & 108.72 & 167.37 & [27] \\
\hline 148 & \multirow{5}{*}{$\begin{array}{c}\text { Prism } \\
40 \mathrm{~mm} \times 40 \mathrm{~mm} \times \\
160 \mathrm{~mm}\end{array}$} & 0.16 & 0.19 & 15.00 & 79.00 & 0.00 & 135.04 & 135.05 & [28] \\
\hline 149 & & 0.16 & 0.19 & 15.00 & 79.00 & 1.00 & 135.04 & 183.29 & [28] \\
\hline 150 & & 0.16 & 0.19 & 15.00 & 79.00 & 2.00 & 135.04 & 190.67 & [28] \\
\hline 151 & & 0.16 & 0.19 & 15.00 & 79.00 & 3.00 & 135.04 & 225.85 & [28] \\
\hline 152 & & 0.16 & 0.19 & 15.00 & 79.00 & 4.00 & 135.04 & 249.68 & [28] \\
\hline 153 & \multirow{10}{*}{$\begin{array}{c}\text { Cylinder } \\
\mathrm{D} \times \mathrm{H} 100 \mathrm{~mm} \times \\
200 \mathrm{~mm}\end{array}$} & 0.13 & 0.20 & 6.00 & 30.00 & 0.00 & 115.13 & 115.13 & [29] \\
\hline 154 & & 0.13 & 0.20 & 6.00 & 30.00 & 1.00 & 115.13 & 134.16 & [29] \\
\hline 155 & & 0.13 & 0.20 & 6.00 & 30.00 & 2.00 & 115.13 & 136.95 & [29] \\
\hline 156 & & 0.13 & 0.20 & 6.00 & 30.00 & 3.00 & 115.13 & 144.94 & [29] \\
\hline 157 & & 0.13 & 0.20 & 6.00 & 30.00 & 4.00 & 115.13 & 151.64 & [29] \\
\hline 158 & & 0.13 & 0.55 & 18.00 & 32.73 & 0.00 & 115.13 & 115.13 & [29] \\
\hline 159 & & 0.13 & 0.55 & 18.00 & 32.73 & 1.00 & 115.13 & 121.22 & [29] \\
\hline 160 & & 0.13 & 0.55 & 18.00 & 32.73 & 2.00 & 115.13 & 117.29 & [29] \\
\hline 161 & & 0.13 & 0.55 & 18.00 & 32.73 & 3.00 & 115.13 & 121.22 & [29] \\
\hline 162 & & 0.13 & 0.55 & 18.00 & 32.73 & 4.00 & 115.13 & 115.03 & [29] \\
\hline
\end{tabular}

Note: * W/B, D, L, AR, VR, PCS and CS denote the water to binder ratio, diameter, length, aspect ratio, volume fraction, and compressive strength of concrete without and with steel fibers. 
Table 2. Experimental Data used for establishing Flexural Strength ANN Model.

\begin{tabular}{|c|c|c|c|c|c|c|c|c|c|}
\hline \multirow{2}{*}{ No. } & \multirow{2}{*}{$\begin{array}{l}\text { Specimens } \\
\text { Dimension }\end{array}$} & \multirow{2}{*}{ W/B } & \multicolumn{4}{|c|}{ Steel Fiber } & \multirow{2}{*}{${ }^{*} P F S$} & \multirow{2}{*}{${ }^{* *} F S$} & \multirow{2}{*}{ Reference } \\
\hline & & & $\mathrm{D} / \mathrm{mm}$ & $L / \mathrm{mm}$ & $A R$ & $V F / \%$ & & & \\
\hline 1 & \multirow{6}{*}{$\begin{array}{c}\text { Prism } \\
40 \mathrm{~mm} \times 40 \mathrm{~mm} \times \\
160 \mathrm{~mm}\end{array}$} & 0.15 & 0.40 & 13.00 & 32.50 & 0.00 & 18.00 & 18.00 & [30] \\
\hline 2 & & 0.17 & 0.40 & 13.00 & 32.50 & 0.20 & 18.00 & 27.50 & [30] \\
\hline 3 & & 0.18 & 0.40 & 13.00 & 32.50 & 0.20 & 18.00 & 22.00 & [30] \\
\hline 4 & & 0.15 & 0.40 & 13.00 & 32.50 & 0.00 & 19.00 & 19.00 & [30] \\
\hline 5 & & 0.17 & 0.40 & 13.00 & 32.50 & 0.20 & 19.00 & 29.00 & [30] \\
\hline 6 & & 0.18 & 0.40 & 13.00 & 32.50 & 0.20 & 19.00 & 22.50 & [30] \\
\hline 7 & \multirow{16}{*}{$\begin{array}{c}\text { Prism } \\
40 \mathrm{~mm} \times 40 \mathrm{~mm} \times \\
160 \mathrm{~mm}\end{array}$} & 0.30 & 0.20 & 13.00 & 65.00 & 0.00 & 10.95 & 10.95 & [9] \\
\hline 8 & & 0.30 & 0.20 & 13.00 & 65.00 & 0.50 & 10.95 & 12.54 & [9] \\
\hline 9 & & 0.30 & 0.20 & 13.00 & 65.00 & 1.00 & 10.95 & 14.55 & [9] \\
\hline 10 & & 0.30 & 0.20 & 13.00 & 65.00 & 2.00 & 10.95 & 16.23 & [9] \\
\hline 11 & & 0.25 & 0.20 & 13.00 & 65.00 & 0.00 & 11.54 & 11.54 & [9] \\
\hline 12 & & 0.25 & 0.20 & 13.00 & 65.00 & 0.50 & 11.54 & 13.51 & [9] \\
\hline 13 & & 0.25 & 0.20 & 13.00 & 65.00 & 1.00 & 11.54 & 15.02 & [9] \\
\hline 14 & & 0.25 & 0.20 & 13.00 & 65.00 & 2.00 & 11.54 & 16.51 & [9] \\
\hline 15 & & 0.20 & 0.20 & 13.00 & 65.00 & 0.00 & 13.97 & 13.97 & [9] \\
\hline 16 & & 0.20 & 0.20 & 13.00 & 65.00 & 0.50 & 13.97 & 15.24 & [9] \\
\hline 17 & & 0.20 & 0.20 & 13.00 & 65.00 & 1.00 & 13.97 & 17.24 & [9] \\
\hline 18 & & 0.20 & 0.20 & 13.00 & 65.00 & 2.00 & 13.97 & 18.40 & [9] \\
\hline 19 & & 0.17 & 0.20 & 13.00 & 65.00 & 0.00 & 15.11 & 18.43 & [9] \\
\hline 20 & & 0.17 & 0.20 & 13.00 & 65.00 & 0.50 & 15.11 & 16.24 & [9] \\
\hline 21 & & 0.17 & 0.20 & 13.00 & 65.00 & 1.00 & 15.11 & 18.11 & [9] \\
\hline 22 & & 0.17 & 0.20 & 13.00 & 65.00 & 2.00 & 15.11 & 19.04 & [9] \\
\hline 23 & \multirow{3}{*}{$\begin{array}{c}\text { Prism } \\
100 \mathrm{~mm} \times 100 \mathrm{~mm} \\
\times 500 \mathrm{~mm} \\
\end{array}$} & 0.24 & 0.20 & 20.00 & 100.00 & 0.00 & 8.23 & 8.23 & [11] \\
\hline 24 & & 0.24 & 0.20 & 20.00 & 100.00 & 1.00 & 8.23 & 7.24 & [11] \\
\hline 25 & & 0.24 & 0.20 & 20.00 & 100.00 & 2.00 & 8.23 & 9.34 & [11] \\
\hline 26 & \multirow{4}{*}{$\begin{array}{l}\text { Prism100 mm } \times \\
100 \mathrm{~mm} \times 500 \mathrm{~mm}\end{array}$} & 0.14 & 0.50 & 30.00 & 60.00 & 0.00 & 9.22 & 9.22 & [12] \\
\hline 27 & & 0.14 & 0.50 & 30.00 & 60.00 & 1.00 & 9.22 & 15.07 & [12] \\
\hline 28 & & 0.14 & 0.50 & 30.00 & 60.00 & 2.00 & 9.22 & 24.57 & [12] \\
\hline 29 & & 0.15 & 0.50 & 30.00 & 60.00 & 3.00 & 9.22 & 29.24 & [12] \\
\hline 30 & \multirow{5}{*}{$\begin{array}{c}\text { Prism100 mm } \times \\
100 \mathrm{~mm} \times 400 \mathrm{~mm}\end{array}$} & 0.20 & 0.20 & 13.00 & 65.00 & 0.00 & 10.13 & 10.13 & [14] \\
\hline 31 & & 0.20 & 0.20 & 13.00 & 65.00 & 0.50 & 10.13 & 12.55 & [14] \\
\hline 32 & & 0.20 & 0.20 & 13.00 & 65.00 & 1.50 & 10.13 & 15.17 & [14] \\
\hline 33 & & 0.20 & 0.20 & 13.00 & 65.00 & 2.50 & 10.13 & 16.51 & [14] \\
\hline 34 & & 0.20 & 0.20 & 13.00 & 65.00 & 3.50 & 10.13 & 20.66 & [14] \\
\hline 35 & \multirow{5}{*}{$\begin{array}{c}\text { Prism } \\
40 \mathrm{~mm} \times 40 \mathrm{~mm} \times \\
160 \mathrm{~mm}\end{array}$} & 0.24 & 0.25 & 13.00 & 52.00 & 0.00 & 11.02 & 11.02 & [15] \\
\hline 36 & & 0.24 & 0.25 & 13.00 & 52.00 & 1.00 & 11.02 & 12.33 & [15] \\
\hline 37 & & 0.24 & 0.25 & 13.00 & 52.00 & 1.50 & 11.02 & 16.51 & [15] \\
\hline 38 & & 0.24 & 0.25 & 13.00 & 52.00 & 2.00 & 11.02 & 19.25 & [15] \\
\hline 39 & & 0.24 & 0.25 & 13.00 & 52.00 & 2.50 & 11.02 & 25.36 & [15] \\
\hline 40 & \multirow{4}{*}{$\begin{array}{c}\text { Prism } \\
40 \mathrm{~mm} \times 40 \mathrm{~mm} \times \\
160 \mathrm{~mm}\end{array}$} & 0.20 & 0.22 & 13.00 & 59.09 & 2.00 & 26.68 & 29.90 & [16] \\
\hline 41 & & 0.20 & 0.22 & 13.00 & 59.09 & 4.00 & 26.68 & 50.63 & [16] \\
\hline 42 & & 0.20 & 0.22 & 13.00 & 59.09 & 5.00 & 26.68 & 73.67 & [16] \\
\hline 43 & & 0.20 & 0.22 & 13.00 & 59.09 & 0.00 & 26.68 & 26.68 & [16] \\
\hline 44 & \multirow{5}{*}{$\begin{array}{c}\text { Prism } \\
40 \mathrm{~mm} \times 40 \mathrm{~mm} \times \\
160 \mathrm{~mm}\end{array}$} & 0.16 & 0.24 & 13.00 & 54.17 & 0.00 & 10.25 & 10.25 & [18] \\
\hline 45 & & 0.16 & 0.24 & 13.00 & 54.17 & 0.50 & 10.25 & 11.38 & [18] \\
\hline 46 & & 0.16 & 0.24 & 13.00 & 54.17 & 1.00 & 10.25 & 20.75 & [18] \\
\hline 47 & & 0.16 & 0.24 & 13.00 & 54.17 & 1.50 & 10.25 & 27.66 & [18] \\
\hline 48 & & 0.16 & 0.24 & 13.00 & 54.17 & 2.00 & 10.25 & 30.38 & [18] \\
\hline 49 & \multirow{4}{*}{$\begin{array}{c}\text { Prism } \\
40 \mathrm{~mm} \times 40 \mathrm{~mm} \times \\
160 \mathrm{~mm}\end{array}$} & 0.17 & 0.16 & 9.00 & 56.25 & 0.00 & 22.60 & 22.60 & [19] \\
\hline 50 & & 0.19 & 0.16 & 9.00 & 56.25 & 1.10 & 22.60 & 25.80 & [19] \\
\hline 51 & & 0.19 & 0.16 & 9.00 & 56.25 & 2.10 & 22.60 & 51.40 & [19] \\
\hline 52 & & 0.19 & 0.16 & 9.00 & 56.25 & 4.20 & 22.60 & 60.30 & [19] \\
\hline
\end{tabular}


Table 2. Cont.

\begin{tabular}{|c|c|c|c|c|c|c|c|c|c|}
\hline \multirow[b]{2}{*}{ No. } & \multirow{2}{*}{$\begin{array}{l}\text { Specimens } \\
\text { Dimension }\end{array}$} & \multirow{2}{*}{ W/B } & \multicolumn{4}{|c|}{ Steel Fiber } & \multirow{2}{*}{${ }^{*}$ PFS } & \multirow{2}{*}{${ }^{* *} F S$} & \multirow{2}{*}{ Reference } \\
\hline & & & $\mathrm{D} / \mathrm{mm}$ & $L / \mathrm{mm}$ & $A R$ & $V F / \%$ & & & \\
\hline 53 & \multirow{12}{*}{$\begin{array}{c}\text { Prism } \\
40 \mathrm{~mm} \times 40 \mathrm{~mm} \times \\
160 \mathrm{~mm}\end{array}$} & 0.20 & 0.22 & 13.00 & 59.09 & 0.00 & 16.60 & 16.60 & [21] \\
\hline 54 & & 0.20 & 0.22 & 13.00 & 59.09 & 0.50 & 16.60 & 17.24 & [21] \\
\hline 55 & & 0.20 & 0.22 & 13.00 & 59.09 & 1.00 & 16.60 & 19.60 & [21] \\
\hline 56 & & 0.20 & 0.22 & 13.00 & 59.09 & 1.50 & 16.60 & 21.30 & [21] \\
\hline 57 & & 0.20 & 0.22 & 13.00 & 59.09 & 2.00 & 16.60 & 26.10 & [21] \\
\hline 58 & & 0.20 & 0.22 & 13.00 & 59.09 & 2.50 & 16.60 & 33.00 & [21] \\
\hline 59 & & 0.20 & 0.22 & 13.00 & 59.09 & 3.00 & 16.60 & 35.20 & [21] \\
\hline 60 & & 0.20 & 0.22 & 13.00 & 59.09 & 3.50 & 16.60 & 36.50 & [21] \\
\hline 61 & & 0.20 & 0.65 & 13.00 & 38.46 & 1.50 & 16.60 & 17.60 & [21] \\
\hline 62 & & 0.20 & 0.65 & 13.00 & 38.46 & 2.00 & 16.60 & 18.50 & [21] \\
\hline 63 & & 0.20 & 0.65 & 13.00 & 38.46 & 2.50 & 16.60 & 19.00 & [21] \\
\hline 64 & & 0.20 & 0.65 & 13.00 & 38.46 & 3.00 & 16.60 & 21.00 & [21] \\
\hline 65 & \multirow{8}{*}{$\begin{array}{c}\text { Prism } \\
100 \mathrm{~mm} \times 100 \mathrm{~mm} \\
\times 400 \mathrm{~mm}\end{array}$} & 0.16 & 0.22 & 13.50 & 61.36 & 0.00 & 17.02 & 17.02 & [25] \\
\hline 66 & & 0.16 & 0.22 & 13.50 & 61.36 & 2.00 & 17.02 & 20.58 & [25] \\
\hline 67 & & 0.16 & 0.22 & 13.50 & 61.36 & 3.00 & 17.02 & 23.36 & [25] \\
\hline 68 & & 0.16 & 0.22 & 13.50 & 61.36 & 4.00 & 17.02 & 18.38 & [25] \\
\hline 69 & & 0.16 & 0.22 & 13.50 & 61.36 & 0.00 & 22.11 & 22.11 & [25] \\
\hline 70 & & 0.16 & 0.22 & 13.50 & 61.36 & 2.00 & 22.11 & 24.10 & [25] \\
\hline 71 & & 0.16 & 0.22 & 13.50 & 61.36 & 3.00 & 22.11 & 21.63 & [25] \\
\hline 72 & & 0.16 & 0.22 & 13.50 & 61.36 & 4.00 & 22.11 & 25.06 & [25] \\
\hline 73 & \multirow{20}{*}{$\begin{array}{c}\text { Prism } \\
100 \mathrm{~mm} \times 100 \mathrm{~mm} \\
\times 400 \mathrm{~mm}\end{array}$} & 0.20 & 0.25 & 14.00 & 56.00 & 0.00 & 14.60 & 14.60 & [26] \\
\hline 74 & & 0.20 & 0.25 & 14.00 & 56.00 & 1.00 & 14.60 & 19.40 & [26] \\
\hline 75 & & 0.20 & 0.25 & 14.00 & 56.00 & 2.00 & 14.60 & 27.10 & [26] \\
\hline 76 & & 0.20 & 0.25 & 14.00 & 56.00 & 3.50 & 14.60 & 35.90 & [26] \\
\hline 77 & & 0.20 & 0.25 & 14.00 & 56.00 & 5.00 & 14.60 & 38.60 & [26] \\
\hline 78 & & 0.20 & 0.25 & 14.00 & 56.00 & 0.00 & 11.50 & 11.50 & [26] \\
\hline 79 & & 0.20 & 0.25 & 14.00 & 56.00 & 1.00 & 11.50 & 17.90 & [26] \\
\hline 80 & & 0.20 & 0.25 & 14.00 & 56.00 & 2.00 & 11.50 & 25.20 & [26] \\
\hline 81 & & 0.20 & 0.25 & 14.00 & 56.00 & 3.50 & 11.50 & 30.40 & [26] \\
\hline 82 & & 0.20 & 0.25 & 14.00 & 56.00 & 5.00 & 11.50 & 31.70 & [26] \\
\hline 83 & & 0.20 & 0.25 & 14.00 & 56.00 & 0.00 & 18.20 & 18.20 & [26] \\
\hline 84 & & 0.20 & 0.25 & 14.00 & 56.00 & 1.00 & 18.20 & 26.30 & [26] \\
\hline 85 & & 0.20 & 0.25 & 14.00 & 56.00 & 2.00 & 18.20 & 31.40 & [26] \\
\hline 86 & & 0.20 & 0.25 & 14.00 & 56.00 & 3.50 & 18.20 & 33.70 & [26] \\
\hline 87 & & 0.20 & 0.25 & 14.00 & 56.00 & 5.00 & 18.20 & 35.60 & [26] \\
\hline 88 & & 0.20 & 0.25 & 14.00 & 56.00 & 0.00 & 19.40 & 19.40 & [26] \\
\hline 89 & & 0.20 & 0.25 & 14.00 & 56.00 & 1.00 & 19.40 & 24.10 & [26] \\
\hline 90 & & 0.20 & 0.25 & 14.00 & 56.00 & 2.00 & 19.40 & 32.80 & [26] \\
\hline 91 & & 0.20 & 0.25 & 14.00 & 56.00 & 3.50 & 19.40 & 38.90 & [26] \\
\hline 92 & & 0.20 & 0.25 & 14.00 & 56.00 & 5.00 & 19.40 & 40.50 & [26] \\
\hline 93 & \multirow{5}{*}{$\begin{array}{c}\text { Prism } \\
100 \mathrm{~mm} \times 100 \mathrm{~mm} \\
\times 400 \mathrm{~mm}\end{array}$} & 0.14 & 0.22 & 13.00 & 59.09 & 0.00 & 10.85 & 10.85 & [27] \\
\hline 94 & & 0.14 & 0.22 & 13.00 & 59.09 & 0.50 & 10.85 & 14.54 & [27] \\
\hline 95 & & 0.14 & 0.22 & 13.00 & 59.09 & 1.00 & 10.85 & 17.03 & [27] \\
\hline 96 & & 0.14 & 0.22 & 13.00 & 59.09 & 1.50 & 10.85 & 19.13 & [27] \\
\hline 97 & & 0.16 & 0.22 & 13.00 & 59.09 & 2.00 & 10.85 & 21.37 & [27] \\
\hline 98 & \multirow{5}{*}{$\begin{array}{c}\text { Prism } \\
40 \mathrm{~mm} \times 40 \mathrm{~mm} \times \\
160 \mathrm{~mm}\end{array}$} & 0.16 & 0.19 & 15.00 & 79.00 & 0.00 & 21.30 & 21.30 & [28] \\
\hline 99 & & 0.16 & 0.19 & 15.00 & 79.00 & 1.00 & 21.30 & 25.40 & [28] \\
\hline 100 & & 0.16 & 0.19 & 15.00 & 79.00 & 2.00 & 21.30 & 32.50 & [28] \\
\hline 101 & & 0.16 & 0.19 & 15.00 & 79.00 & 3.00 & 21.30 & 41.60 & [28] \\
\hline 102 & & 0.16 & 0.19 & 15.00 & 79.00 & 4.00 & 21.30 & 45.20 & [28] \\
\hline
\end{tabular}

Note: * PFS and FS denotes the flexural strength of concrete without and with steel fibers, respectively, which is tested by 3-point bending test. ** The specimens for flexural strength testing varies in sizes, due to lack of proper standards. According to GB/T 31387 [46], prism specimen of $100 \times 100 \times 300 \mathrm{~mm}$ is regarded as standard for homogeneity of data. Researches shows that the size effects have no significant in flexural strength and the convert coefficient between standard and other size specimens can be taken as 1.0 [48].

Therefore, a total of 166 compressive strength experimental data sets and 102 flexural strength experimental data sets were obtained to establish the training-testing database. Among the data, $80 \%$ (133 and 80) data sets were selected as training sets, and the remaining data sets ( $20 \%$ of the total data) 
were used for testing, respectively. The testing data were selected randomly to eliminate the effects of artificial selection on the results. In accordance with existing calculation models [31-38], the water to binder ratio $(W / B)$, the diameter $(D)$, the length $(L)$, the aspect ratio $(A R)$, and the volume fraction $(V F)$ of steel fibers, and the compressive strength (PCS) or the flexural strength (PFS) of UHPFRC without steel fibers were selected as the main input parameters, and the compressive strength (CS) or flexural strength (FS) of UHPFRC was used as the output variable. Table 3 shows the ranges of the input and the output variables in the compressive strength database and the flexural strength database in this study.

Table 3. Ranges of Parameters in Compressive Strength and Flexural Strength Data base.

\begin{tabular}{ccccc}
\hline \multirow{2}{*}{ Variables } & \multicolumn{2}{c}{ Compressive Strength } & \multicolumn{2}{c}{ Flexural Strength } \\
\cline { 2 - 5 } & Minimum & Maximum & Minimum & Maximum \\
\hline$W / B$ & 0.13 & 0.30 & - & - \\
$D / \mathrm{mm}$ & 0.15 & 0.65 & 0.16 & 0.65 \\
$L / \mathrm{mm}$ & 6 & 30 & 9 & 30 \\
$A R$ & 100 & 30 & 32.5 & 100 \\
$V F / \%$ & 0.00 & 5.00 & 0.00 & 5.00 \\
$P C S / \mathrm{MPa}$ & 72.15 & 220.98 & 8.23 & 26.88 \\
$C S / \mathrm{MPa}$ & 72.15 & 249.68 & 8.23 & 73.67 \\
\hline
\end{tabular}

\subsection{Proposed ANN Model}

The ANN models proposed in this paper have three layers: an input layer, a hidden layer, and an output layer (in Figure 1). The number of input and output nodes are determined by the parameters of the research problems. However, because of reliable mathematical formulas for calculating the number of hidden layer nodes are not available, the initial number of hidden layer nodes should be estimated by trials. The Log-Sigmoid activation function is used in the hidden layer, and the linear activation function is used in the output layer.

To develop the ANN model for predicting the flexural strength and the compressive strength of UHPFRC, a series of trials were carried out in order to determine the number of layers and other parameters of the ANN models by the minimum mean square error (MSE) of the training data. The length $(L)$, the diameter $(D)$, the aspect ratio $(A R)$, and the volume fraction $(V F)$ of steel fibers and the flexural strength (PFS) of UHPFRC without fibers are investigated as inputs, and the flexural strength (FS) of UHPFRC is as output, meanwhile, the length $(L)$, the diameter $(D)$, the aspect ratio $(\mathrm{AR})$, and the volume fraction $(V F)$ of steel fibers, the water to binder ratio $(W / B)$ and the compressive strength (PCS) of UHPFRC without fibers are investigated as inputs, and the compressive strength (CS) of UHPFRC is as output. The parameters of the ANN models for predicting the flexural strength and the compressive strength of UHPFRC are shown in Table 4 and the structures of models are shown in Figure 2.

Table 4. Parameters used in the ANN models.

\begin{tabular}{ccc}
\hline Parameters & Flexural Strength Model & Compressive Strength Model \\
\hline Number of input layer nodes & 5 & 6 \\
Number of hidden layers & 1 & 1 \\
Number of hidden layer nodes & 15 & 20 \\
Number of output layer nodes & 1 & 1 \\
Momentum factor & 0.8 & 0.6 \\
Learning rate & 0.3 & 0.3 \\
Target error & 0.00001 & 0.00001 \\
Learning cycle & 10,000 & 10,000 \\
\hline
\end{tabular}


(a)

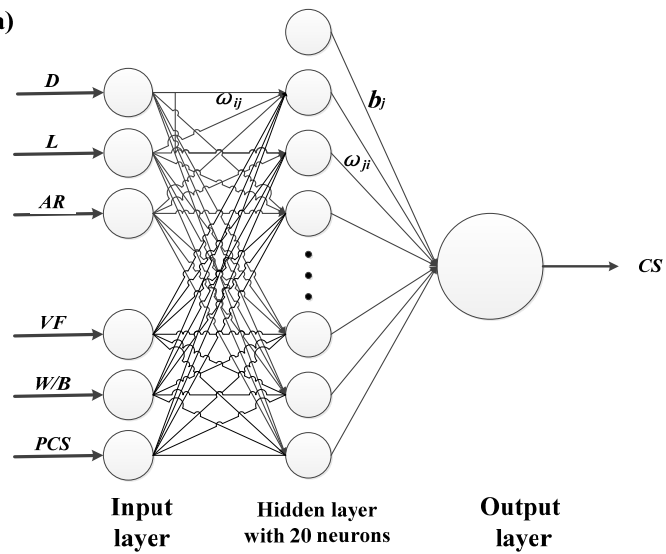

(b)

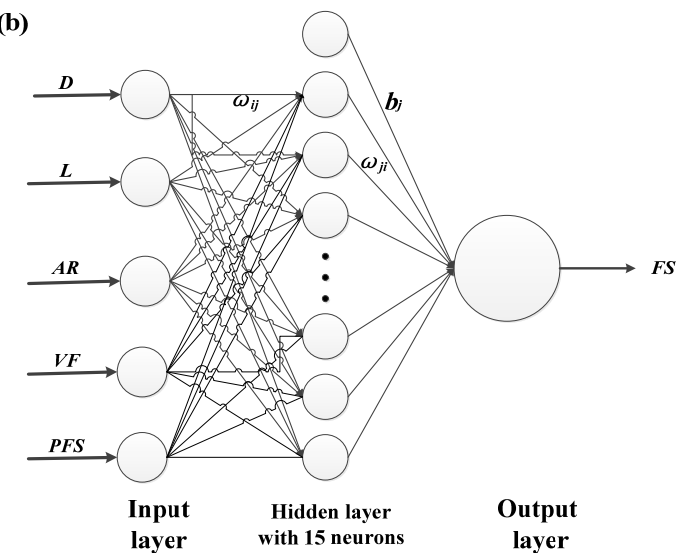

Figure 2. Structure of ANN models, (a) Compressive strength ANN model, (b) Flexural strength ANN model; W/B (water to binder ratio); PCS (compressive strength of UHPFRC without fibers); VF (volume fractions); AR (aspect ratio); PFS (flexural strength of UHPFRC without fibers); CS (compressive strength of UHPFRC); FS (flexural strength of UHPFRC). In (a), the compressive strength ANN model developed in this study has three layers with six neurons in input layer, twenty neurons in hidden layer and one neural in output layer; in (b), the compressive strength ANN model that was developed in this study has three layers with five neurons in input layer, fifteen neurons in hidden layer, and one neural in output layer. In these two figures, $\omega_{i j}$ is the weight between the $i_{t h}$ neuron in the input layer and the $j_{t h}$ neuron in the hidden layer, $\omega_{j i}$ is the weight between the $i_{t h}$ neuron in the hidden layer and the $j_{t h}$ neuron in the output layer, and $b_{j}$ is the bias of the $j_{t h}$ layer in the output layer.

\subsection{Processing Data}

The collected data applied to develop the ANN models should be normalized within the specific limits to eliminate the non-singular data, improve the precision of results, accelerate the convergence speed, and reduce the calculation time. The majority of normalization expressions are linear or logarithmic functions [43]. In this paper, a sample function, as expressed in Equation (10), was adopted to normalize the data.

$$
X_{i, n o r m}=0.1+0.8 \times\left(X_{i}-X_{\min }\right) /\left(X_{\max }-X_{\min }\right)
$$

where $X_{i, \text { norm }}$ is normalized data and $X_{\max }$ and $X_{\min }$ are the maximum and minimum value of data, respectively. An inverse normalized process is applied to the output layer to get the test data.

\section{Results and Discussion}

\subsection{Results Assessment Criteria}

A successfully trained ANN model should give an accurate output prediction, not only for input data used in the training process, but also for new testing data unfamiliar to the model within the range of the training database. In this study, six indicators were applied in order to evaluate the performance of the compressive strength ANN model and five indicators for the flexural strength ANN model, respectively. These three indicators are root mean square error $(R M S)$, absolute fraction of variance $\left(R^{2}\right)$, and integral absolute error $(I A E)$, which are calculated by Equations (11)-(13), respectively [38,45]. Theoretically, When the RMS and the IAE tend to zero and the $R^{2}$ tends to one, the proposed models predict the experimental data accurately.

$$
R M S=\sqrt{\frac{1}{n} \sum_{k=1}^{n}\left(t_{k}-o_{k}\right)^{2}}
$$




$$
\begin{gathered}
R^{2}=1-\left[\sum_{i=1}^{n}\left(t_{i}-o_{i}\right)^{2} / \sum_{i=1}^{n} o^{2}\right] \\
I A E=\left(\left(\sum_{k=1}^{n}\left(o_{k}-t_{k}\right)^{2}\right)^{1 / 2}\right) /\left(\sum_{k=1}^{n} o_{k}\right) \times 100 \%
\end{gathered}
$$

where $n$ is the total number of data; $t_{k}$ is the target value of $j_{t h}$ data; $o_{k}$ is the output value of $j_{t h}$ data; and, $\bar{o}$ is the average value of output value.

\subsection{Results Evaluation}

\subsubsection{Predicting Model for Compressive Strength}

The compressive strength ANN model developed in this study was adopted to evaluating the effects of steel fibers on the compressive strength of UHPFRC. Comparisons between the predicted and the experimental values for the training and testing data of the compressive strength ANN model were shown in Figure 3. It was evident that the predicted values from the training and testing data, as calculated by the ANN model, were closed to the target values. This phenomenon demonstrated that the ANN model could successfully learn the nonlinear relationship between the input and output variables. Therefore, the ANN model possessed potential in estimating the effects of steel fibers on the compressive strength of UHPFRC.
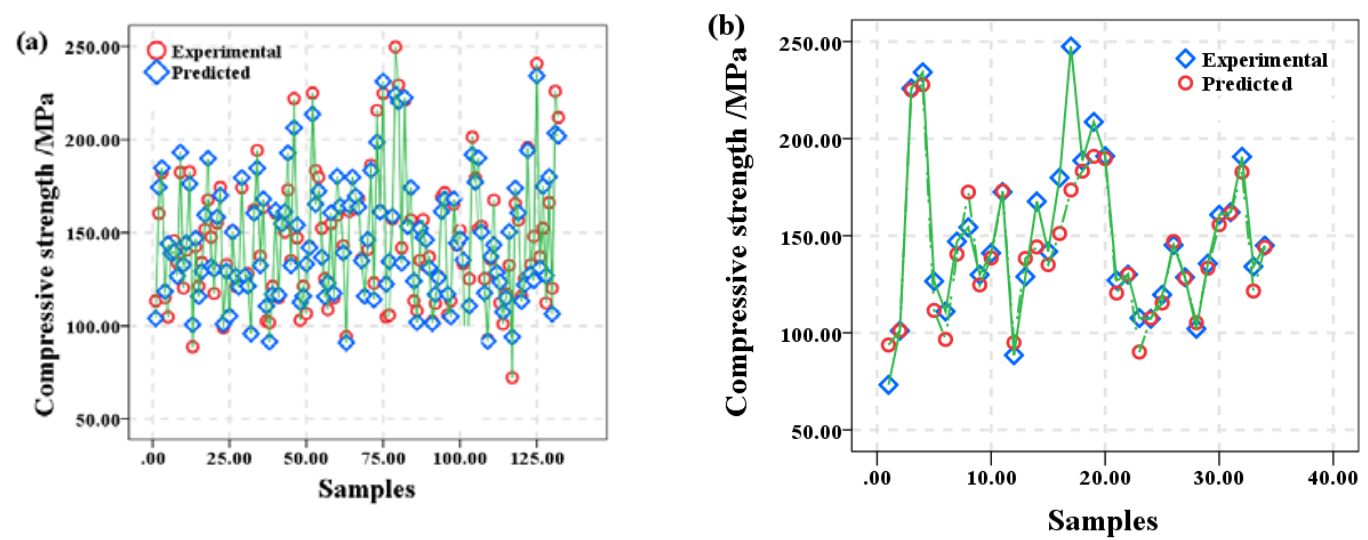

Figure 3. Comparison of predicted values from compressive strength ANN model with experimental results: (a) Training data, (b) Testing data. The figures show that the comparison between the target values and predicted values of testing and training data from experiments and compressive strength ANN model, the horizontal axis denotes number of training or testing data, and the vertical axis denotes the compressive strength. It was obvious that the predicted values accord with the target values.

The input layer weights (ILW), input layer bias (ILB), hidden layer weights (HLW), and hidden layer bias (HLB) of the compressive strength ANN model are given by Equations (14)-(17), respectively. 


$$
\mathrm{ILW}=\left[\begin{array}{cccccc}
1.5393 & -0.2127 & -0.0696 & -1.4878 & 0.2019 & 0.9149 \\
0.4685 & 0.8877 & -0.0434 & 1.2903 & -1.2371 & 1.0505 \\
0.9528 & -0.4423 & 0.3086 & 1.0429 & 0.6903 & -1.7290 \\
1.0738 & 0.4441 & -1.5045 & 0.4403 & 0.5282 & -1.1052 \\
-0.3691 & 0.5837 & 0.7369 & -1.7350 & 1.1595 & 0.0825 \\
-0.8850 & -1.3132 & 1.1989 & 1.2757 & -0.6270 & -1.0193 \\
0.5121 & 0.5669 & 1.4762 & 1.6028 & -0.0071 & -0.7542 \\
-1.1525 & -0.3260 & -0.5873 & 1.3268 & -1.2193 & -0.4579 \\
0.1460 & -0.7018 & -0.5530 & -0.1367 & -0.1684 & -2.0212 \\
0.5865 & -0.3549 & 0.1205 & 2.1009 & 0.9361 & -0.8912 \\
0.8419 & -1.6760 & 0.4490 & 0.3753 & -1.4686 & 1.2215 \\
1.0630 & -1.0899 & -0.5704 & -1.4019 & 1.1772 & 0.5639 \\
1.3647 & -0.8022 & -0.9620 & -0.6018 & -0.8654 & 0.0328 \\
0.8870 & -0.7126 & 1.1355 & -0.8674 & 1.3638 & 0.9118 \\
0.3813 & -0.7026 & -1.6491 & -2.4456 & -0.4511 & -0.7444 \\
0.3807 & -1.1086 & -1.0983 & 1.5312 & 0.5930 & 0.8901 \\
0.7989 & 0.5241 & -1.0421 & -0.9141 & 0.8343 & 1.2352 \\
-2.5278 & -0.8382 & -0.7985 & 0.4525 & -0.3999 & 0.4810 \\
-1.6123 & 0.2212 & 0.7569 & -0.5385 & -0.1606 & -1.2508 \\
2.6458 & 1.4649 & 0.8273 & 0.0796 & -0.1915 & -0.5157
\end{array}\right]
$$

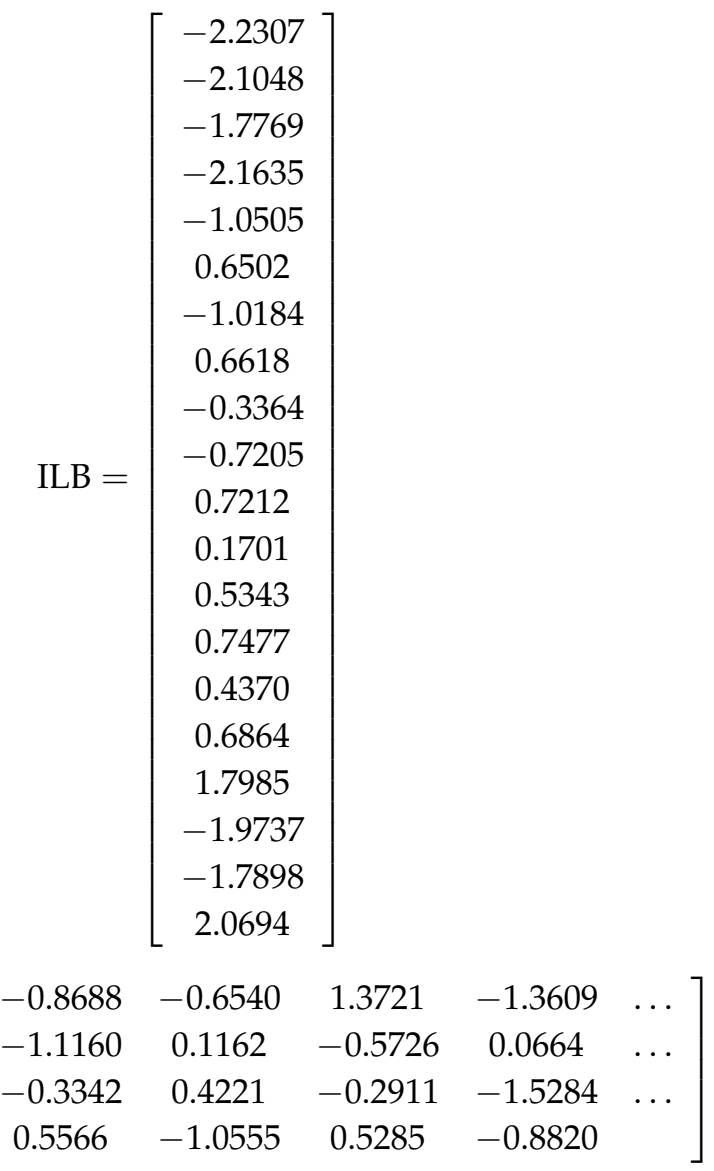

$$
\begin{aligned}
& \mathrm{HLB}=[-0.6960]
\end{aligned}
$$

The performance indicators of the compressive strength ANN model for training and testing data, including $R M S, R^{2}$, and IAE, were listed in Table 5. In Table 5, the indicators show that the proposed 
model produced good results. Thus, the ANN model studied in this paper had high-precision and it was suitable for predicting the compressive strength of UHPFRC.

Table 5. The indicators of training and testing of Compressive strength ANN model.

\begin{tabular}{ccc}
\hline Indicators & Training & Testing \\
\hline$R M S$ & 0.0876 & 0.0980 \\
$R^{2}$ & 0.9923 & 0.9901 \\
$I A E$ & 0.0005 & 0.0019 \\
\hline
\end{tabular}

\subsubsection{Prediction Model for Flexural Strength}

The flexural strength the ANN model that was developed in this study was adopted to predict the flexural strength of UHPFRC. Comparisons between the predicted and experimental values for the training and testing data of the ANN model were shown in Figure 4. It was evident that the predicted values from the training and the testing data, as calculated by the ANN model, were closely to the target values. This phenomenon demonstrated that the ANN model could successfully learn the nonlinear relationship between the input and output variables. Therefore, the ANN model possessed potential to estimate the effects of steel fibers on the flexural strength of UHPFRC.
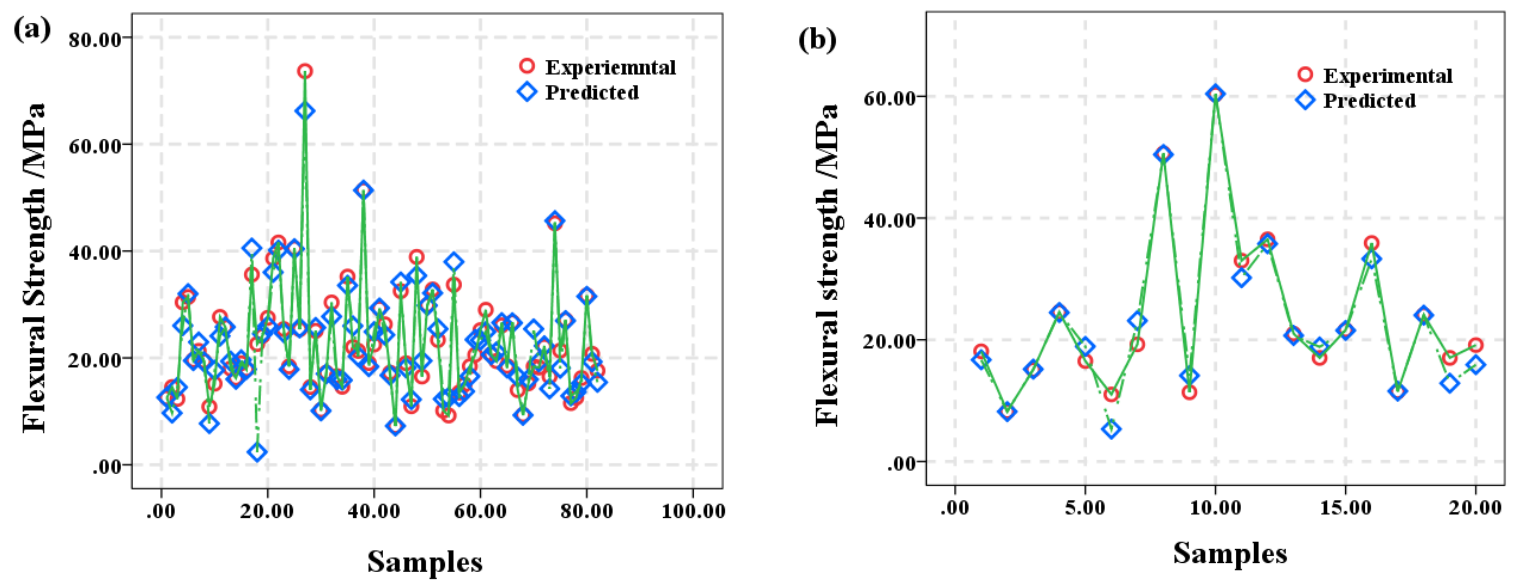

Figure 4. Comparison of predicted values from flexural strength ANN model with experimental results: (a) Training data, (b) Testing data. The figures showed that the comparison between the target values and predicted values of testing and training data from experiments and flexural strength ANN model, the horizontal axis denotes number of training or testing data and the vertical axis denotes the flexural strength. It was obvious that the predicted values accord with the target values.

The input layer weights (ILW), input layer bias (ILB), hidden layer weights (HLW), and hidden layer bias (HLB) of the flexural strength ANN model are given by Equations (18)-(21), respectively. 


$$
\mathrm{ILW}=\left[\begin{array}{ccccc}
-1.6618 & -0.1478 & -0.2359 & 0.3162 & 1.6211 \\
-0.9701 & 0.8838 & -0.8155 & -1.0247 & 0.8334 \\
2.0128 & -1.2745 & -0.6188 & -0.7977 & -0.5454 \\
1.5968 & 0.7523 & -0.0592 & 0.0347 & -1.6347 \\
0.1462 & -1.3740 & 1.5325 & -0.5921 & -2.0480 \\
-1.7972 & 0.6656 & 0.6023 & 1.6868 & -0.6638 \\
-0.3699 & -0.5348 & 0.3489 & -1.7462 & -0.1823 \\
-1.0360 & -1.0720 & -1.1409 & -1.4832 & -0.7843 \\
0.5253 & 0.6616 & 2.7165 & -1.4818 & 0.0997 \\
1.5109 & 1.9836 & 0.8304 & -1.1222 & 0.6302 \\
1.7022 & -2.0969 & -1.8292 & -1.1385 & -0.8313 \\
-1.2578 & 0.9580 & 0.3124 & -0.0636 & -1.2783 \\
0.8136 & 0.4598 & -0.4208 & 2.2028 & -1.5568 \\
1.9101 & -0.0758 & 0.5641 & -0.3651 & -2.0535 \\
1.4933 & 0.4085 & -0.9748 & -0.5404 & -1.6774
\end{array}\right]
$$

The performance indicators of the flexural strength ANN model for training and testing data, including $R M S, R^{2}$, and IAE, were listed in Table 6. In Table 6, the indicators show that the proposed model produced good results. Thus, the flexural strength of the ANN model studied in this paper had high-precision and was suitable for evaluating the effects of steel fibers on the flexural strength of UHPFRC.

Table 6. The indicators of Training and Testing of Flexural strength ANN model.

\begin{tabular}{ccc}
\hline Indicators & Training & Testing \\
\hline$R M S$ & 0.1492 & 0.0376 \\
$R^{2}$ & 0.9777 & 0.9986 \\
$I A E$ & 0.0011 & 0.0004 \\
\hline
\end{tabular}




\subsection{Comparison with Other Models}

\subsubsection{Compressive Strength Models}

To study the reliability of the proposed model, the results from the compressive strength ANN model were compared with the values that were calculated by existing models presented in the literature [34-36]. There are rarely models for predicting the compressive strength of UHPFRC, thus, the models predicting the compressive strength of steel fiber reinforced concrete (SFRC) are summarized in Table 7. In these models, the Nataraja model is utilized to predict the compressive strength (30-50 MPa) of SFRC, the VF ranges from 0.5 to $1.0 \%$ and the AR are 55 and 82; the Ezeldin model is applied to predict the compressive strength (35-85 MPa) of SFRC, the VF ranges from $30 \mathrm{~kg} / \mathrm{m}^{3}$ to $60 \mathrm{~kg} / \mathrm{m}^{3}$ and the AR are 60, 75, and 100; Yuchen Qu model is applied to predict the compressive strength (30-50 MPa) of SFRC, the VF ranges from 0 to 3.4\%, and the AR are from 50 to 100.

Table 7. Analytical models for predicting the compressive strength of steel fiber reinforced concrete (SFRC).

\begin{tabular}{cc}
\hline Analytical Model & Compressive Strength \\
\hline Nataraja [34] & $f_{c f}=f_{c}+2.1604\left(R I_{w}\right)$ \\
Ezeldin [35] & $f_{c f}=f_{c}+3.51\left(R I_{w}\right)$ \\
Yuchen Qu [36] & $f_{c f}=f_{c}+2.35\left(R I_{v}\right)$ \\
\hline
\end{tabular}

Where $f_{c f}$ and $f_{c}$ are the compressive strength of SFRC and plain concrete, respectively; $R I_{w}$ and $R I_{v}$ are the reinforcing indexes of steel fibers weight fraction and steel fibers volume fraction, respectively, $R I_{w}$ is around 3.25 times of $R I_{v}, R I_{w}=w_{f} \times l_{f} / d_{f}, w_{f}$ is the weight fraction of steel fibers, $l_{f}$ is the length of steel fibers, and the $d_{f}$ is the diameter of steel fibers.

The analytical models that are described above were applied to calculate the compressive strength of UHPFRC based on the collected data. Table 8 illustrates the mean value, standard deviation $(S D)$, and IAE of $f_{\text {predicted }} / f_{\text {exp erimental }}$ for the analytical models of previous studies and the compressive strength ANN model of this study. In Table 9, the mean values for the analytical models were less than one, ranging from 0.8830 to 0.9454 , and the $S D$ and the IAE were within the ranges of $0.1191-0.1268$ and 1.03-1.34\%, respectively. The mean values from the ANN model in this study were closely to one, and the $S D$ and the IAE were quite low. Figure 5 indicates that the predicted values obtained from the analytical models of previous studies might underestimate the experimental results with high variation. The ranges of predicted results relative to experimental results that were obtained from the Nataraja model, Ezeldin model, and Yuchen Qu model are -45-15\%, -40-30\%, and -50-10\%, respectively. Whereas, the range of predicted results relative to experimental results obtained from ANN model is $-15-15 \%$. In the comparison with the analytical models for predicting the compressive strength of UHPFRC, the ANN model that was proposed in this study could predict the behaviors of UHPFRC very well. Thus, the predictions of the compressive strength ANN model are considerably accuracy and stability for evaluating the effects of steel fibers on the compressive strength of UHPFRC.

Table 8. The Mean value, standard deviation (SD), and integral absolute error (IAE) of ANN model and analytical models.

\begin{tabular}{cccc}
\hline Models & Mean & SD & IAE \\
\hline ANN model & 1.0050 & 0.0896 & $0.70 \%$ \\
Nataraja model & 0.9152 & 0.1191 & $1.13 \%$ \\
Ezeldin model & 0.9454 & 0.1216 & $1.03 \%$ \\
Yuchen Qu model & 0.8830 & 0.1268 & $1.34 \%$ \\
\hline
\end{tabular}


Table 9. Analytical models for predicting the Flexural strength of SFRC.

\begin{tabular}{cc}
\hline Analytical Model & Flexural Strength \\
\hline JGJ/T 221 [31] & $f_{f f}=f_{f}\left(1+1.25 V_{f} l_{f} / d_{f}\right)$ \\
Swamy [32] & $f_{f f}=0.97 f_{f}\left(1-V_{f}\right)+3.41 V_{f} l_{f} / d_{f}$ \\
Won-Kya Chai [33] & $f_{f f}=f_{f}+0.103 V_{f} l_{f} / d_{f}$ \\
\hline
\end{tabular}

(a)
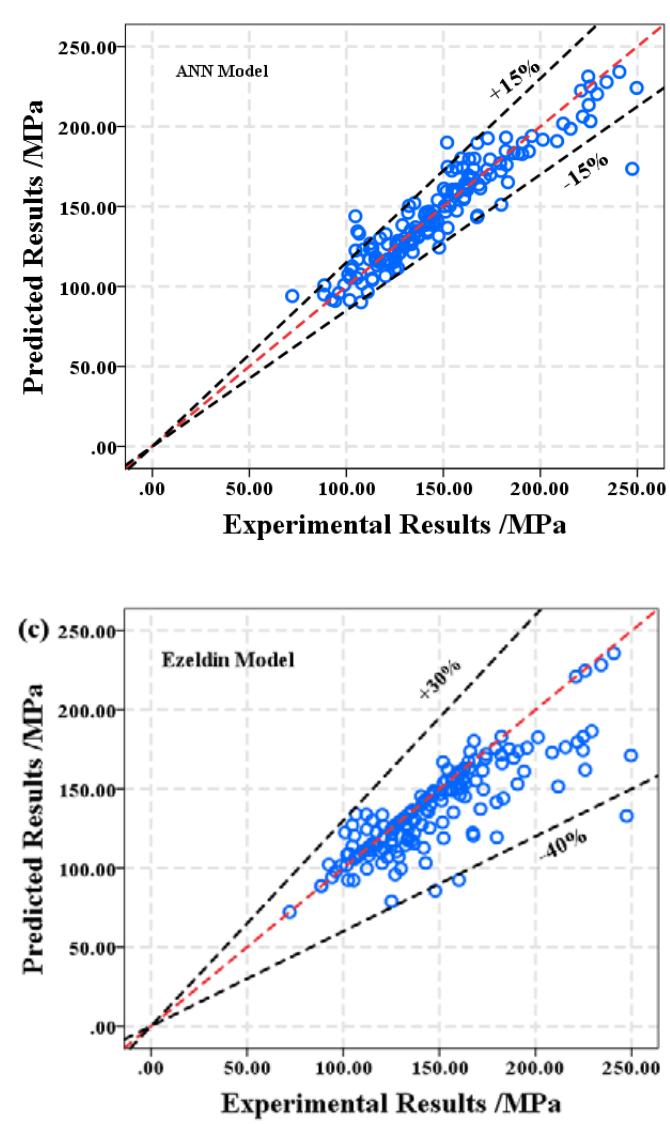
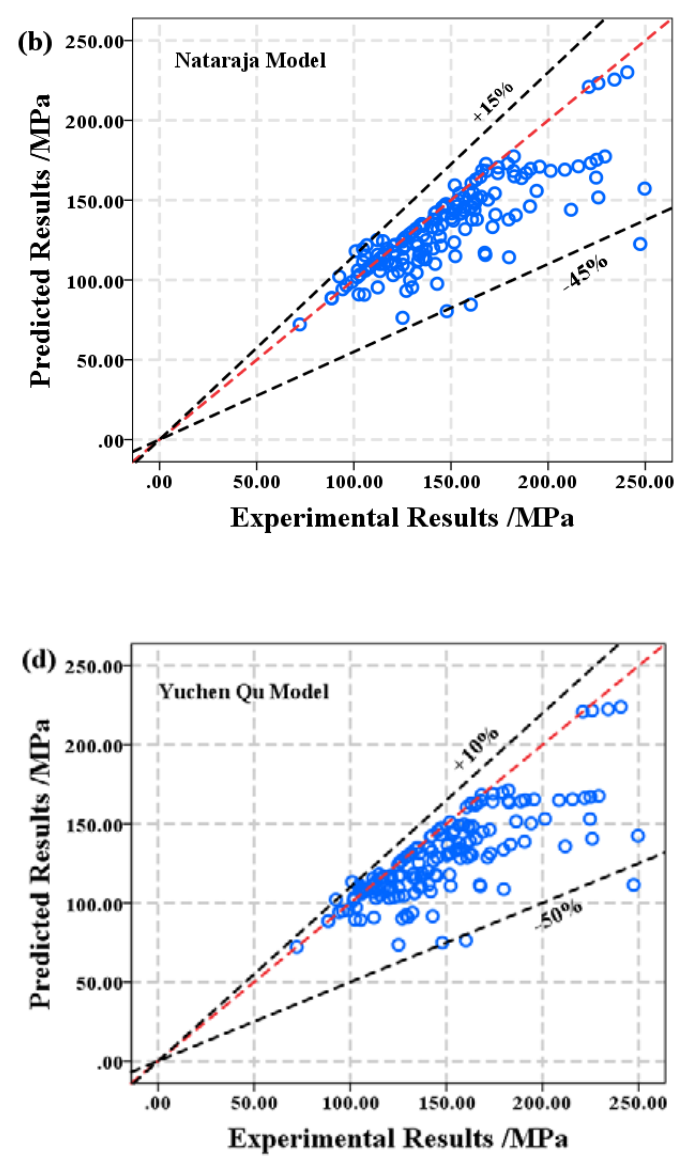

Figure 5. Comparison between predicted values and experimental values from three analytical models (proposed by Nataraja, Ezeldin, and Yuchen Qu) in previous studies and the ANN model studied in this paper. The 45 degree diagonal line (red line) denotes the predicted values equal to the experimental values and the black lines denote that the maximum ranges between predicted results and experimental results.

\subsubsection{Flexural Strength Models}

To study the reliability of the proposed model, the results from the flexural strength ANN model were compared with the values that were calculated by existing models presented in the literature [31-33]. There are rarely models for predicting the flexural strength of UHPFRC, thus, the models predicting the flexural strength of steel fiber reinforced concrete (SFRC) are summarized in Table 9.

Where $f_{f f}$ and $f_{f}$ are the flexural strength of steel fiber reinforced concrete and plain concrete, respectively; $V_{f}$ is the volume fraction of steel fibers, $l_{f}$ is the length of steel fibers, and the $d_{f}$ is the diameter of steel fibers.

The analytical models that are described above were applied to calculate the flexural strength of UHPFRC based on the collected data. Table 10 illustrates the mean value, standard deviation $(S D)$, and IAE of $f_{\text {predicted }} / f_{\text {exp erimental }}$ for the analytical models of previous studies and the flexural 
strength ANN model of this study. In Table 10, the mean values for the analytical models ranged from 0.8429 to 1.1458 , and the $S D$ and the $I A E$ were within the ranges of $0.2055-0.4431$ and $3.03-4.04 \%$, respectively. The mean values from the ANN model in this study were fairly close to one, and the $S D$ and the $I A E$ were quite low. Figure 6 indicates that the predicted values that were obtained from the analytical models of previous studies might underestimate the experimental results with high variation. The ranges of predicted results relative to experimental results obtained from JGJ/T 221 model, Swamy model, and Won-Kya Chai model are $-35-180 \%,-50-30 \%$, and $-35-150 \%$, respectively. Whereas, the range of predicted results relative to experimental results obtained from ANN model is $-15-15 \%$. In the comparison with the analytical models of flexural strength for UHPFRC, the ANN model that was proposed in this study could predict the flexural behavior of UHPFRC very well. Thus, the predictions of the flexural strength ANN model are considerably accuracy and stability for evaluating the effects of steel fibers on the flexural strength of UHPFRC.

Table 10. The Mean value, SD, and IAE of ANN model and analytical models.

\begin{tabular}{cccc}
\hline Models & Mean & SD & IAE \\
\hline ANN model & 0.9915 & 0.1509 & $1.50 \%$ \\
JGJ/T 221 model & 1.2807 & 0.4431 & $4.04 \%$ \\
Swamy model & 0.8429 & 0.2055 & $3.03 \%$ \\
Won-Kya Chai model & 1.1458 & 0.3547 & $3.30 \%$ \\
\hline
\end{tabular}

(a)

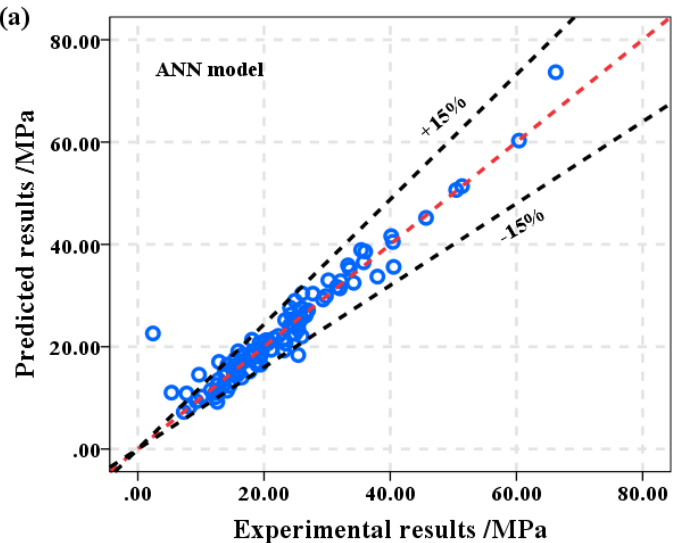

(c)

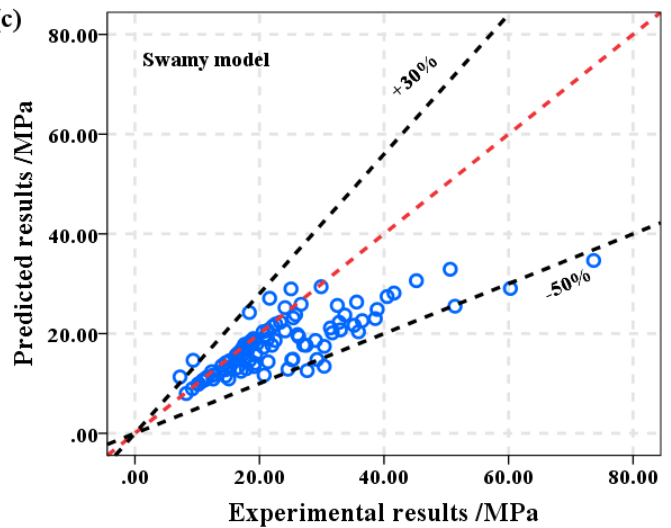

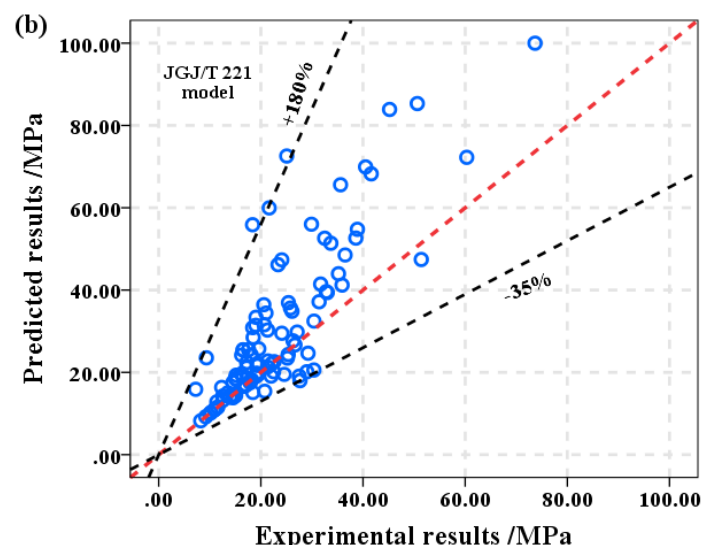

(d)

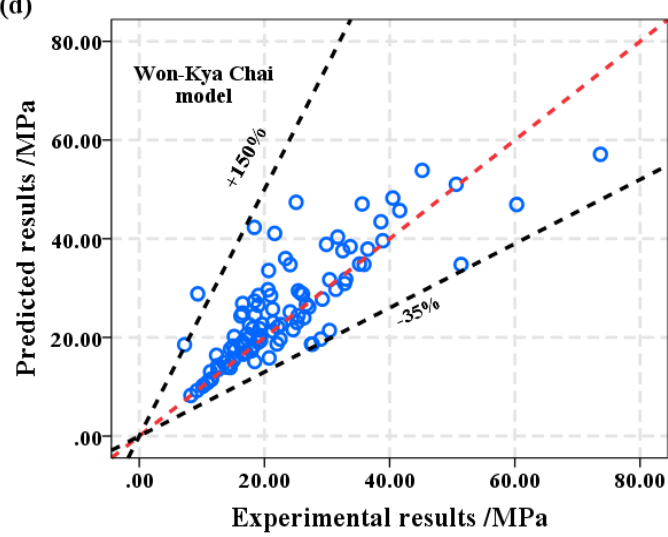

Figure 6. Comparison between predicted values and experimental values from three analytical models (proposed by JGJ/T 221, Swamy, Won-Kya Chai) in previous studies and the ANN model studied in this paper. The 45 degree diagonal line (red line) denotes the predicted values equal to the experimental values and the black lines denote that the maximum ranges between predicted results and experimental results. 


\section{Conclusions}

In this paper, the ANN method was applied to evaluate the compressive strength and the flexural strength of UHPFRC. Two reliable database, consisting of 166 compressive strength data sets and 102 flexural strength data sets from previous literature, were established, of which, 133 and 80 samples were randomly chosen for training and the remainder for testing to establish the compressive strength ANN model and flexural strength ANN model, respectively. The conclusions were as follows:

(1) The compressive strength ANN model was trained by using the LM algorithm, with twenty neurons in hidden layers, revealing great prediction performance. The predicted values were fairly close to the experimental results for both the training and testing data sets in the proposed model.

(2) The flexural strength ANN model was trained by using the LM algorithm, with twenty neurons in hidden layers, revealing great prediction performance. The predicted values were fairly close to the experimental results for both the training and testing data sets in the proposed model.

(3) The results that were obtained from the compressive strength ANN model were compared with three analytical models proposed in other studies. The comparison indicated that the analytical models proposed by others may underestimate the compressive strength by approximately $10 \%$ on average, whereas the predicted values from the ANN model in this study agree with the experimental values.

(4) The results obtained from the flexural strength ANN model were compared with three analytical models that were proposed in other studies. The comparison indicated that the analytical models proposed by others may varied from 0.8429 to 1.1458 on average values, whereas the predicted values from the ANN model in this study agree with the experimental values.

(5) The ANN models that were proposed in this study have high applicability and reliability with respect to evaluating the effects of steel fibers on the compressive strength and the flexural strength of UHPFRC.

\section{Research Limitations}

In this paper, the authors only focus on the effects of some properties of steel fibers on the compressive strength and the flexural strength of UHPFRC, but these properties of steel fibers have not been analyzed separately. Besides, other properties of steel fibers, such as fiber brand, fiber strength, fiber shapes, and so on, are not considered in this paper. This subject will be further studied.

Author Contributions: D.X. collected the data sets, X.C. analyzed the data; W.C. proposed the methods and wrote the paper.

Funding: This research was funded by the International cooperation project fund of the Ministry of science and technology, grant number 2014DFR81000.

Conflicts of Interest: The authors declare no conflicts of interest.

\section{References}

1. Zdeb, T. An analysis of the steam curing and autoclaving process parameters for reactive powder concretes. Construct. Build. Mater. 2017, 131, 758-766. [CrossRef]

2. Grünewald, S. Performance-Based Design of Self-Compacting Fiber Reinforced Concrete; Delft University of Technology: Delft, The Netherlands, 2004.

3. Yu, R.; Spiesz, P.; Brouwers, H.J.H. Mix design and properties assessment of Ultra-High Performance Fiber Reinforced Concrete. Cem. Concr. Res. 2014, 56, 29-39. [CrossRef]

4. Zhao, L.P.; Gao, D.Y.; Zhu, H.T. Effects of steel fibers on the strength and the ductility of concrete. J. North Chin. Inst. Water Conserv. Hydroelectr. Power 2012, 33, 29-32.

5. Ortega, J.M.; Sánchez, I.; Climent, M.A. Impedance spectroscopy study of the effect of environmental conditions in the microstructure development of OPC and slag cement mortars. Materials 2017, 10, 569-583. [CrossRef] 
6. Sánchez, I. Influence of environment on durability of fly ash cement mortars. ACI Mater. J. 2012, 109, 647-656.

7. Joshaghani, A.; Balapour, M.; Ramezanianpour, A.A. Effects of controlled environmental conditions on mechanical, microstructural and durability properties of cement mortar. Construct. Build. Mater. 2018, 164, 134-149. [CrossRef]

8. Ramezanianpour, A.A.; Malhotra, V.M. Effects of curing on the compressive strength resistance to chloride-ion penetration and porosity of concrete incorporating slag, fly ash or silica fume. Cem. Concr. Compos. 1995, 17, 125-133. [CrossRef]

9. Bae, B.; Choi, H.K.; Choi, C.S. Correlation between tensile strength and compressive strength of ultra-high strength concrete reinforced with steel fiber. J. Korea Concr. Inst. 2015, 27, 253-263. [CrossRef]

10. Prem, P.R.; Bharatkumar, B.H.; Murthy, A.R. Influence of curing regime and steel fibers on the mechanical properties of UHPC. Mag. Concr. Res. 2015, 67, 988-1002. [CrossRef]

11. Ayira, O.J.F. Investigating the properties of reactive powder concrete (RPC) compressive and flexural strength. Diss. Bachelor Eng. 2013, 4, 9758-9762.

12. Khalil, W.; Damha, L.S. Mechanical properties of reactive powder concrete with various steel fiber and silica fume contents. ACTA Tecnia Corviniensis Bull. Eng. 2014, 7, 47-58.

13. Guo, J. Influence of compressive strength of reactive powder concrete with different fibers. Concrete 2016, 5, 87-90.

14. Ma, K.; Que, A.; Liu, C. Impact analysis of fibers on mechanical properties of reactive powder concrete. Concrete 2016, 3, 76-83.

15. Zhong, S.; Wang, Y.; Gao, H. Effect of fibers on strength of self-compacting reactive powder concrete. J. Build. Mater. 2008, 11, 522-527.

16. Wang, Q.; Guo, Z.; Xiang, Z.; Shao, J. Experimental research on proportion of reactive powder concrete 200 (RPC200). J. Arch. Civil Eng. Depart. 2007, 1, 70-74.

17. Huang, L.; Xing, F.; Deng, L.; Huang, P. Study on factors affecting the strength of reactive powder concrete. J. Shenzhen Univ. Sci. Eng. 2004, 21, 178-182.

18. Bi, Q.; Yang, Z.; Jiao, Q.; Wang, H. Experiment study on mechanical properties of a hubrid fiber reinforced reactive powder concrete. J. DaLian Jiaotong Univ. 2009, 30, 19-21.

19. Zhang, P.; Kang, Q.; Shen, Z.; Wang, Z. Experimental research on the mechanical properties of steel fiber and carbon fiber reinforce RPC. Funct. Mater. 2010, 233-235.

20. Wang, X.; Wang, Y. Mechanical properties of RPC with different steel fiber volume contents. J. Build. Mater. 2015, 18, 941-945.

21. Wang, J.; Hao, X.; Ji, F. Effects of steel fibers on the mechanical properties of reactive powder concrete. Low Temp. Arch. Technol. 2018, 3, 18-20.

22. Jia, F.; AN, M.; Zhang, H.; Yu, Z. Effect of fibers on bond properties between steel bar and reactive powder concrete. J. Build. Mater. 2012, 15, 847-851.

23. Zhang, Q.; Wei, Y.; Zhang, J.; Feng, P. Influence of steel fiber content on fracture properties of RPC. J. Build. Mater. 2014, 17, 24-29.

24. Zeng, J.; Wu, Y.; Lin, Q. Researches on the compressive mechanics properties of steel fiber RPC. J. Fuzhou Univ. Nat. Sci. 2005, 33, 132-137.

25. Cao, X.; Peng, J.; LI, W. Study on mechanics properties of reactive powder concrete with different fibers. Chin. Concr. Cem. Product. 2014, 10, 54-57.

26. Guo, T.; Teng, T.; Yu, Q. Influence of steel fibers contents on the strength and ductility of RPC. City House 2016, 23, 119-121.

27. Jia, F.; Wang, W.; He, K.; Xia, Y.; Wang, J.; Zha, Y.; Kong, D. Study on basic properties of reactive powder concrete with different fiber. Chin. Concr. Cem. Product. 2016, 9, 53-56.

28. Wang, Z.; Wang, J.; Yuan, J. Study on the Aggregates and mix Proportion of RPC. In Proceedings of the 13th National Academic Conference on Concrete and Prestressed Concrete, Beijing, China, 1 January 2016; pp. 342-347.

29. Al-Tikrite, A.; Hadi, M.N.S. Mechanical properties of reactive powder concrete containing industrial and waste steel fibers at different ratios under compression. Construct. Build. Mater. 2017, 154, 1024-1034. [CrossRef]

30. Maroliya, M.K. An investigation on reactive powder concrete containing steel fiber and fly ash. Int. J. Eng. Technol. Adv. Eng. 2012, 2, 538-545. 
31. Ministry of Housing and Urban-Rural Development of the People's Republic of China (MOHURD). JGJ/T 221-2010: Technical Specification for Application of Fiber Reinforced Concrete; China Ministry of Construction, China Architecture \& Building Press: Beijing, China, 2002.

32. Swamy, R.N.; Mangat, P.S. A theory for the flexural strength of steel fiber reinforced concrete. Cem. Concr. Res. 1974, 4, 313-325. [CrossRef]

33. Choi, W.K. An experimental study on the flexural strength of fiber reinforced concrete structure. Int. J. Saf. 2012, 11, 26-28.

34. Nataraja, M.C.; Dhang, N.; Gupda, A.P. Stress-strain curves for steel fiber reinforced concrete under compression. Cem. Concr. Compos. 1999, 21, 383-390. [CrossRef]

35. Ezeldin, A.S.; Balaguru, P.N. Normal and high strength fiber reinforced concrete under compression. J. Mater. Civil Eng. 1992, 4, 415-429. [CrossRef]

36. Ou, Y.C.; Tsai, M.S.; Liu, K.; Chang, K.C. Compressive behavior of steel fiber-reinforced concrete with a high index. J. Mater. Civil Eng. 2012, 24, 207-215. [CrossRef]

37. Nehdi, M.L.; Soliman, A.M. Artificial intelligence model for early-age autogenous shrinkage of concrete. ACI Mater. J. 2012, 109, 353-362.

38. Cascardi, A.; Micelli, F.; Aiello, M.A. An artificial neural networks model for the prediction of the compressive strength of FRP-confined concrete circular columns. Eng. Struct. 2017, 140, 199-208. [CrossRef]

39. Açikgenç, M.; Ulaş, M.; Alyamaç, K.E. Using an Artificial Neural Network to Predict Mix Compositions of Steel Fiber-Reinforced Concrete. Arab. J. Sci. Eng. 2015, 40, 407-419. [CrossRef]

40. Vidivelli, B.; Jayaranjini, A. Prediction of compressive strength of high performance concrete containing industrial by products using artificial neural networks. Int. J. Civil Eng. Technol. 2016, 7, 302-314.

41. Altun, F.; Kişi, Ö.; Aydin, K. Predicting the compressive strength of steel fiber added lightweight concrete using neural network. Comput. Mater. Sci. 2008, 42, 259-265. [CrossRef]

42. Zealakshmi, D.; Ravichandran, A.; Kothandaraman, S. Prediction of Flexural Performance of Confined Hybrid Fibre Reinforced High Strength Concrete Beam by Artificial Neural Networks. Ind. J. Sci. Technol. 2016, 9, 1-6. [CrossRef]

43. Sobhani, J.; Najimi, M.; Pourkhorshidi, A.; Parhizkar, T. Prediction of the compressive strength of no-slump concrete: A comparative study of regression, neural network and ANFIS models. Construct. Build. Mater. 2010, 24, 709-718. [CrossRef]

44. Golafshani, E.M.; Rahai, A.; Sebt, M.H.; Akbarpour, H. Prediction of bond strength of spliced steel bars in concrete using artificial neural network and fuzzy logic. Construct. Build. Mater. 2012, 36, 411-418. [CrossRef]

45. Sarıdemir, M. Predicting the compressive strength of mortars containing metakaolin by artificial neural networks and fuzzy logic. Adv. Eng. Softw. 2009, 40, 920-927. [CrossRef]

46. Alshihri, M.M.; Azmy, A.M.; El-Bisy, M.S. Neural networks for predicting compressive strength of structural light weight concrete. Construct. Build. Mater. 2009, 23, 2214-2219. [CrossRef]

47. China Standardization Administration. GB/T 31387-2015: Code for Reactive Powder Concrete, China Standardization Administration; China Architecture and Building Press: Beijing, China, 2015.

48. Mansur, M.; Islam, M. Interpretation of concrete strength for nonstandard specimens. J. Mater. Civil Eng. 2002, 14, 151-155. [CrossRef]

49. Lu, X.; Wang, Y.; Fu, C.; Zheng, W. Basic mechanical properties indexes of reactive powder concrete. J. Harbin Inst. Techanol. 2014, 46, 1-10.

50. Benjamin, G.; Marshall, D. Cylinder or sube: Strengtrhtesting of 80 to $200 \mathrm{MPa}$ (11.6 to $29 \mathrm{ksi})$ ultra-high-performance fiber-reinforced concrete. J. ACI Mater. 2008, 105, 603-609.

51. Kusumawardaningsih, Y.; Fehling, E.; Ismail, M. UHPC compressive strength test specimens: Cylinder or cube. Procedia Eng. 2015, 125, 1076-1080. [CrossRef]

(C) 2018 by the authors. Licensee MDPI, Basel, Switzerland. This article is an open access article distributed under the terms and conditions of the Creative Commons Attribution (CC BY) license (http://creativecommons.org/licenses/by/4.0/). 